\section{A) Check for updates}

Cite this: Dalton Trans., 2021, 50 14400

Received 1st September 2021 Accepted 14th September 2021 DOI: $10.1039 / \mathrm{d} 1 \mathrm{dt} 02948 \mathrm{~g}$ rsc.li/dalton

\title{
Tin(Iv) fluoride complexes with neutral phosphine coordination and comparisons with hard $\mathrm{N}$ - and O-donor ligands $\uparrow$
}

\author{
Rhys P. King, ${ }^{\text {a }}$ Madeleine S. Woodward, (D) a Julian Grigg, ${ }^{\text {b }}$ Graeme McRobbie, ${ }^{b}$ \\ William Levason (D) ${ }^{a}$ and Gillian Reid (D) *a
}

\begin{abstract}
The reactions of trans- $\left[\mathrm{SnF}_{4}\left(\mathrm{PMe}_{3}\right)_{2}\right]$ with one, two or three equivalents of $\mathrm{Me}_{3} \mathrm{SiO}_{3} \mathrm{SCF}_{3}$ (TMSOTF), respectively, in anhydrous $\mathrm{CH}_{2} \mathrm{Cl}_{2}$ form six-coordinate $\left[\mathrm{SnF}_{4-n}\left(\mathrm{PMe}_{3}\right)_{2}(\mathrm{OTf})_{n}\right](n=1-3)$, which have been characterised by microanalysis, IR and multinuclear NMR $\left({ }^{1} \mathrm{H},{ }^{19} \mathrm{~F}\left\{{ }^{1} \mathrm{H}\right\},{ }^{31} \mathrm{P}\left\{{ }^{1} \mathrm{H}\right\}\right.$ and $\left.{ }^{119} \mathrm{Sn}\right)$ spectroscopy. The crystal structure of $\left[\mathrm{SnF}_{3}\left(\mathrm{PMe}_{3}\right)_{2}(\mathrm{OTf})\right]$ reveals the three fluorines are in a mer-arrangement with mutually trans $\mathrm{PMe}_{3}$ ligands. The multinuclear NMR spectra confirm this structure is retained in solution, and show that $\left[\mathrm{SnF}_{2}\left(\mathrm{PMe}_{3}\right)_{2}(\mathrm{OTf})_{2}\right]$ has trans-phosphines, while $\left[\mathrm{SnF}\left(\mathrm{PMe}_{3}\right)_{2}(\mathrm{OTf})_{3}\right]$ has trans $\mathrm{PMe}_{3}$ groups and hence mer-triflate ligands. The $\left[\mathrm{SnF}_{4-n}\left(\mathrm{PMe}_{3}\right)_{2}(\mathrm{OTf})_{n}\right]$ are unstable in solution and the decomposition products include $\left[\mathrm{Me}_{3} \mathrm{PF}\right]^{+}$and the tin(II) complexes $\left[\mathrm{Sn}\left(\mathrm{PMe}_{3}\right)_{2}(\mathrm{OTf})_{2}\right]$ and $\left[\mathrm{Sn}_{3} \mathrm{~F}_{5}(\mathrm{OTf})\right]$, both of the latter identified by their crystal structures. The reaction of trans- $\left[\mathrm{SnF}_{4}\left(\mathrm{P}^{\mathrm{i}} \mathrm{Pr}_{3}\right)_{2}\right]$ containing the bulkier phosphine, with one and two equivalents of TMSOTf produced unstable mono- and bis-triflates, which the NMR data also suggest contain weakly coordinated triflate, $\left[\mathrm{SnF}_{3}\left(\mathrm{PPr}_{3}\right)_{2}(\mathrm{OTf})\right]$ and $\left[\mathrm{SnF}_{2}\left(\mathrm{PiPr}_{3}\right)_{2}(\mathrm{OTf})_{2}\right]$, again with axial phosphines, although some OTf dissociation from the former to give $\left[\mathrm{SnF}_{3}\left(\mathrm{P}^{\mathrm{i}} \mathrm{Pr}_{3}\right)_{2}\right]^{+}$may occur in solution at room temperature. The new phosphine complexes of $\mathrm{SnF}_{4}$, trans- $\left[\mathrm{SnF}_{4}\left(\mathrm{P}^{\mathrm{i}} \mathrm{Pr}_{3}\right)_{2}\right]$ and (cis) [SnF $\mathrm{K}_{4} \mathrm{\kappa}^{2}$-triphos)] (triphos $\left.=\mathrm{CH}_{3} \mathrm{C}\left(\mathrm{CH}_{2} \mathrm{PPh}_{2}\right)_{3}\right)$ have also been fully characterised, including the crystal structure of $\left[\mathrm{SnF}_{4}\left(\mathrm{~K}^{2}\right.\right.$-triphos $\left.)\right]$. Attempts to promote $\mathrm{P}_{3}$-coordination by further treatment of this complex with TMSOTf were unsuccessful. The $\left[\mathrm{SnF}_{4}(\mathrm{~L})_{2}\right](\mathrm{L}=$ dmso, py, pyNO, DMF, OPPh $)$ complexes, which exist as mixtures of cis and trans isomers, react with one equivalent of TMSOTf, followed by addition of one equivalent of $\mathrm{L}$, to form the ionic $\left[\mathrm{SnF}_{3}(\mathrm{~L})_{3}\right][\mathrm{OTf}]$ complexes, which were characterised by microanalysis, IR and multinuclear NMR spectroscopy. In nitromethane solution they are a mixture of mer and fac isomers based upon multinuclear NMR data $\left({ }^{1} \mathrm{H},{ }^{19} \mathrm{~F}\left\{{ }^{1} \mathrm{H}\right\},{ }^{119} \mathrm{Sn}\right)$. Reaction of $\left[\mathrm{SnF}_{4}\left(\mathrm{OPPh}_{3}\right)_{2}\right]$ with two equivalents of TMSOTf and further OPPh 3 produced $\left[\mathrm{SnF}_{2}\left(\mathrm{OPPh}_{3}\right)_{4}\right][\mathrm{OTf}]_{2}$, which is a mixture of cis and trans isomers in solution. The crystal structure of $\left[\mathrm{SnF}_{2}\left(\mathrm{OPPh}_{3}\right)_{4}\right][\mathrm{OTf}]_{2}$ confirms the trans isomer in the solid state, with the triflate ionic. These complexes are rare examples of fluorotin(iv) cations with neutral monodentate ligands.
\end{abstract}

\section{Introduction}

In addition to their intrinsic interest, coordination complexes of the main group elements have attracted considerable interest in recent years for a variety of applications, including Lewis

\footnotetext{
${ }^{a}$ School of Chemistry, University of Southampton, Southampton SO17 1BJ, UK. E-mail: G.Reid@soton.ac.uk

${ }^{b}$ GE Healthcare, Pollards Wood, Nightingales Lane, Chalfont St Giles, Bucks, HPS 4 SP, UK

$\dagger$ Electronic supplementary information (ESI) available: Multinuclear NMR and IR spectra associated with each of the new compounds described, together with the crystal structure of $\mathrm{Sn}_{3} \mathrm{~F}_{5}(\mathrm{OTf})$, the X-ray crystallographic parameters. CCDC 2104490-2104493, 2104976, 2104984 and 2106812. For ESI and crystallographic data in CIF or other electronic format see DOI: 10.1039/d1dt02948g
}

acid based and metal free catalysis, development of precursors for the chemical vapour deposition of semiconductor thin films and, particularly in the case of main group fluoride complexes, towards new types of ${ }^{18} \mathrm{~F}$ carriers for positron emission tomography imaging in medicine. ${ }^{1}$

The tin(Iv) halides, $\mathrm{SnX}_{4}(\mathrm{X}=\mathrm{Cl}, \mathrm{Br}$ or I) are tetrahedral monomers that are widely used as Lewis acids and as synthons for tin(Iv) complexes. ${ }^{2-5}$ The complexes are generally formed by adding two neutral donor groups to form six-coordinate cisor trans-[$\left[\mathrm{SnX}_{4}(\mathrm{~L})_{2}\right]$ or cis-[ $\left[\mathrm{SnX}_{4}(\right.$ bidentate) $]$. Only very rarely do neutral ligands displace a coordinated halide to form $\mathrm{Sn}(\mathrm{Iv})$ cations; exceptions are provided by the macrocycles 1,4,7-trimethyl-1,4,7-triazacyclononane, 1,3,5-trimethyl-1,3,5triazacyclohexane and 1,4,7-trithiacyclononane $\left(\mathrm{L}_{3}\right)$, which generate the 'self-ionisation' complexes, $\left[\operatorname{SnX}_{3}\left(\mathrm{~L}_{3}\right)\right]_{2}\left[\operatorname{SnX}_{6}\right]$ 
$(\mathrm{X}=\mathrm{Cl}$ or $\mathrm{Br}){ }^{6,7}$ although more flexible acyclic polydentate ligands, such as $\mathrm{MeC}\left(\mathrm{CH}_{2} \mathrm{AsMe}_{2}\right)_{3}$, behave as bidentate ligands, as in $\left[\mathrm{SnX}_{4}\left\{\kappa^{2}-\mathrm{MeC}\left(\mathrm{CH}_{2} \mathrm{AsMe}_{2}\right)_{3}\right\}\right] .{ }^{8}$ Formation of tin (Iv) cations can be achieved by using halide abstractors such as $\mathrm{AlCl}_{3}, \mathrm{CF}_{3} \mathrm{SO}_{3} \mathrm{SiMe}_{3}$ (TMSOTf) or $\mathrm{Na}\left[\mathrm{BAr}^{\mathrm{F}}\right]\left(\mathrm{BAr}^{\mathrm{F}}=[\mathrm{B}\{3,5-\right.$ $\left.\left.\left.\left(\mathrm{CF}_{3}\right)_{2}\left(\mathrm{C}_{6} \mathrm{H}_{3}\right)\right\}_{4}\right]^{-}\right) \cdot{ }^{9,10}$

Tin(Iv) fluoride contains six-coordinate tin in vertex sharing octahedra $^{11}$ and although readily hydrolysed, is unreactive towards many neutral ligands. As a result of the lack of commercial availability and this limited reactivity, its coordination chemistry was little explored until recently; only one crystal structure was reported (for the complex $\left[\operatorname{SnF}_{4}\left(2,2^{\prime}\right.\right.$-bipy)]) and spectroscopic data were limited. ${ }^{12-17}$ A key development was the synthesis of $\left[\mathrm{SnF}_{4}(\mathrm{MeCN})_{2}\right]$ from $\mathrm{SnF}_{2}$ and $\mathrm{I}_{2}$ in MeCN by Tudela. ${ }^{18}$ The other product, $\mathrm{SnI}_{4}$, does not form a stable MeCN adduct and is readily removed by washing with $\mathrm{CS}_{2}$. The MeCN is easily displaced from $\left[\mathrm{SnF}_{4}(\mathrm{MeCN})_{2}\right]$ by neutral ligands, and hence this is a valuable synthon for developing wider coordination chemistry of $\mathrm{SnF}_{4}$ with a range of ligands. Detailed studies of the reactions of $\left[\mathrm{SnF}_{4}(\mathrm{MeCN})_{2}\right]$ with phosphine and arsine oxides produced a series of complexes $\left[\mathrm{SnF}_{4}\left(\mathrm{R}_{3} \mathrm{EO}\right)_{2}\right](\mathrm{R}=\mathrm{Me}, \mathrm{Ph} ; \mathrm{E}=\mathrm{P}, \mathrm{As})$ and $\left[\mathrm{SnF}_{4}(\mathrm{~L}-\mathrm{L})\right](\mathrm{L}-\mathrm{L}=(\mathrm{L}-\mathrm{L}$ $=o-\mathrm{C}_{6} \mathrm{H}_{4}\left(\mathrm{P}(\mathrm{O}) \mathrm{Ph}_{2}\right)_{2}, \quad o-\mathrm{C}_{6} \mathrm{H}_{4}\left(\mathrm{P}(\mathrm{O}) \mathrm{Me}_{2}\right)_{2}$ or $\mathrm{Ph}_{2} \mathrm{P}(\mathrm{O}) \mathrm{CH}_{2} \mathrm{P}(\mathrm{O})$ $\left.\mathrm{Ph}_{2}\right),{ }^{19}$ and comparison of spectroscopic and structural data on these and their heavier $\mathrm{SnX}_{4}(\mathrm{X}=\mathrm{Cl}, \mathrm{Br}, \mathrm{I})$ analogues confirmed $\mathrm{SnF}_{4}$ as the strongest Lewis acid of the four. Subsequently, these studies were extended to tertiary phosphine complexes, although arsine ligands did not form isolable complexes with $\mathrm{SnF}_{4}$ (in contrast to their behaviour with other $\left.\mathrm{SnX}_{4}\right) .{ }^{8}$ Further studies of N- and O-donor complexes of $\mathrm{SnF}_{4}$ were also included, but none of the ligands showed any tendency to displace fluoride from $\mathrm{SnF}_{4}{ }^{8}$ Fluorotin cations were obtained with the tetradentate $\mathrm{N}$-donor ligand, tris(1ethyl-benzoimidazol-2-ylmethyl)amine $\left(\mathrm{BIMEt}_{3}\right)$ by reaction of $\mathrm{SnF}_{4}, \mathrm{BIMEt}_{3}$ and appropriate amounts of TMSOTf, when $\left[\mathrm{SnF}_{4-x}\left(\mathrm{BIMe}_{3}\right)\right][\mathrm{OTf}]_{x}(x=1,2,3)$ were isolated. ${ }^{20}$ Other fluorotin(Iv) cations appear unknown. ${ }^{12}$

Here we report the reactions of phosphine complexes of tin (Iv) tetrafluoride with some halide abstractors, and several cationic $\mathrm{N}$ - and $\mathrm{O}$-donor ligand complexes, $\left[\mathrm{SnF}_{4-x} \mathrm{~L}_{2+x}\right]^{x+}$, prepared by abstraction of fluoride from $\left[\mathrm{SnF}_{4} \mathrm{~L}_{2}\right]$ with TMSOTf in the presence of added L.

\section{Experimental}

All complex syntheses were carried out using standard Schlenk and vacuum line techniques. Samples were handled and stored in a glove box under a dry dinitrogen atmosphere to exclude moisture. TMSOTf was obtained from Sigma-Aldrich and distilled before use. Tin(II) fluoride was obtained from Alfa Aesar and used as received. Phosphine ligands, $\mathrm{OPPh}_{3}$ and pyNO were obtained from Sigma-Aldrich or Strem and used as received. $\mathrm{Na}\left[\mathrm{BAr}^{\mathrm{F}}\right]$ was made as described. ${ }^{10} \mathrm{CH}_{2} \mathrm{Cl}_{2}, \mathrm{DMF}$, dmso and MeCN were dried by distillation from $\mathrm{CaH}_{2}$, py, diethyl ether and $n$-hexane from Na. $\left[\mathrm{SnF}_{4}(\mathrm{MeCN})_{2}\right]$ was made from $\mathrm{SnF}_{2}$ and $\mathrm{I}_{2}$ in $\mathrm{MeCN}$ as described by Tudela. $^{18}$ $\left[\mathrm{SnF}_{4}\left(\mathrm{PMe}_{3}\right)_{2}\right],{ }^{8}\left[\mathrm{SnF}_{4}(\mathrm{Py})_{2}\right]^{8}$ and $\left[\mathrm{SnF}_{4}\left(\mathrm{OPPh}_{3}\right)_{2}\right]^{19}$ were made by literature methods.

Infrared spectra were recorded as Nujol mulls between CsI plates using a Perkin Elmer Spectrum 100 spectrometer over the range 4000-200 $\mathrm{cm}^{-1} \cdot{ }^{1} \mathrm{H},{ }^{19} \mathrm{~F}\left\{{ }^{1} \mathrm{H}\right\},{ }^{31} \mathrm{P}\left\{{ }^{1} \mathrm{H}\right\}$, and ${ }^{119} \mathrm{Sn}$ NMR spectra were recorded from $\mathrm{CH}_{2} \mathrm{Cl}_{2} / \mathrm{CD}_{2} \mathrm{Cl}_{2}$ or $\mathrm{CH}_{3} \mathrm{NO}_{2}$ / $\mathrm{CD}_{3} \mathrm{NO}_{2}$ solutions using a Bruker AV400 spectrometer and referenced to TMS via the residual solvent resonance, $\mathrm{CFCl}_{3}$, $85 \% \mathrm{H}_{3} \mathrm{PO}_{4}$, or neat $\mathrm{SnMe}_{4}$, as appropriate. Typically, [Cr $\left.(\text { acac })_{3}\right]$ was added as a relaxation agent when recording the ${ }^{119} \mathrm{Sn}$ spectra. Microanalyses were undertaken by London Metropolitan University or Medac.

\section{$\left[\mathrm{SnF}_{3}\left(\mathrm{PMe}_{3}\right)_{2}(\mathrm{OTf})\right]$}

To a solution of $\left[\mathrm{SnF}_{4}\left(\mathrm{PMe}_{3}\right)_{2}\right](0.100 \mathrm{~g}, 0.288 \mathrm{mmol})$ in $\mathrm{CH}_{2} \mathrm{Cl}_{2}$ $(2 \mathrm{~mL})$ a solution of TMSOTf $(0.064 \mathrm{~g}, 0.288 \mathrm{mmol})$ in $\mathrm{CH}_{2} \mathrm{Cl}_{2}$ ( $2 \mathrm{~mL}$ ) was added dropwise to form a clear solution. The reaction was stirred for $2 \mathrm{~h}$, the volatiles were then removed in vacuo to leave a solid, which was washed with hexane $(3 \times 10 \mathrm{~mL})$ and dried in vacuo to form a white powder. Crystals suitable for single crystal X-ray diffraction were grown by layering a $\mathrm{CH}_{2} \mathrm{Cl}_{2}$ solution of the complex with hexane. Yield $0.083 \mathrm{~g}, 60 \%$. Required for $\mathrm{C}_{7} \mathrm{H}_{18} \mathrm{~F}_{6} \mathrm{P}_{2} \mathrm{~S}_{2} \mathrm{Sn}$ (476.91): C, 17.6; H, 3.8. Found: C, 17.5; H, 4.2\%. IR (Nujol/cm $\left.{ }^{-1}\right): \nu=517 \mathrm{~m}, 546 \mathrm{~m}, 573 \mathrm{~m}(\mathrm{Sn}-\mathrm{F})$, $1156\left(-\mathrm{OSO}_{2}\right), 1224,1261\left(\mathrm{CF}_{3}\right) .{ }^{1} \mathrm{H}$ NMR $\left(\mathrm{CD}_{2} \mathrm{Cl}_{2}, 298 \mathrm{~K}\right): \delta=$ $1.70\left(\mathrm{~d},{ }^{2} J_{\mathrm{PH}}=12 \mathrm{~Hz}, \mathrm{CH}_{3}\right) \cdot{ }^{19} \mathrm{~F}\left\{{ }^{1} \mathrm{H}\right\} \mathrm{NMR}\left(\mathrm{CD}_{2} \mathrm{Cl}_{2}, 298 \mathrm{~K}\right): \delta=$ -78.8 (s, OTf), -126.9 (br s, Sn-F), -149.2 (br s, Sn-F); $\left(\mathrm{CD}_{2} \mathrm{Cl}_{2}\right.$, $183 \mathrm{~K}): \delta=-78.5(\mathrm{~s},[3 \mathrm{~F}], \mathrm{OTf}),-127.9\left(\mathrm{td},[2 \mathrm{~F}],{ }^{2} J_{\mathrm{PF}(\text { cis-OTf })}=153\right.$ $\mathrm{Hz},{ }^{2} J_{\mathrm{FF}}=41 \mathrm{~Hz},{ }^{1} J^{119} \mathrm{SnF}=3253 \mathrm{~Hz},{ }^{1} J_{117 \mathrm{SnF}}=3108 \mathrm{~Hz}, \mathrm{Sn}-$ $\left.\mathrm{F}_{\text {cis-OTf }}\right),-151.4\left(\mathrm{tt},[\mathrm{F}],{ }^{2} J_{\mathrm{PF}(\text { trans-OTf })}=121 \mathrm{~Hz},{ }^{2} J_{\mathrm{FF}}=41,{ }^{1} J_{119} \mathrm{SnF}=\right.$ $\left.3013 \mathrm{~Hz},{ }^{1} J_{117 \mathrm{SnF}}=2893 \mathrm{~Hz}, \mathrm{Sn}-\mathrm{F}_{\text {trans-OTf }}\right) .{ }^{31} \mathrm{P}\left\{{ }^{1} \mathrm{H}\right\} \mathrm{NMR}\left(\mathrm{CH}_{2} \mathrm{Cl}_{2}\right.$, $298 \mathrm{~K}): \delta=-6.01(\mathrm{~s}) ;\left(\mathrm{CH}_{2} \mathrm{Cl}_{2}, 183 \mathrm{~K}\right): \delta=-3.2\left(\mathrm{td},{ }^{2} J_{\mathrm{PF}(\text { cis-OTf })}=\right.$ $\left.153 \mathrm{~Hz} ;{ }^{2} J_{\mathrm{PF} \text { (trans-OTf) }}=121 \mathrm{~Hz} ;{ }^{1} J_{119 \mathrm{SnP}}=3412 \mathrm{~Hz} ;{ }^{1} J_{117 \mathrm{SnP}}=3263\right)$. ${ }^{119} \mathrm{Sn}$ NMR $\left(\mathrm{CH}_{2} \mathrm{Cl}_{2}, 183 \mathrm{~K}\right): \delta=-599.9(\mathrm{~m})$.

\section{$\left[\mathrm{SnF}_{2}\left(\mathrm{PMe}_{3}\right)_{2}(\mathrm{OTf})_{2}\right]$}

To a solution of $\left[\mathrm{SnF}_{4}\left(\mathrm{PMe}_{3}\right)_{2}\right](0.100 \mathrm{~g}, 0.288 \mathrm{mmol})$ in $\mathrm{CH}_{2} \mathrm{Cl}_{2}$ (2 mL) a solution of TMSOTf (0.128 g, $0.576 \mathrm{mmol})$ in $\mathrm{CH}_{2} \mathrm{Cl}_{2}$ ( $2 \mathrm{~mL}$ ) was added dropwise to form a clear solution. The reaction was stirred for $2 \mathrm{~h}$. The volatiles were then removed in vacuo to leave a solid, which was washed with hexane $(3 \times$ $10 \mathrm{~mL}$ ) and dried in vacuo to form a white powder. Yield 0.103, 59\%. Required for $\mathrm{C}_{8} \mathrm{H}_{18} \mathrm{~F}_{8} \mathrm{O}_{6} \mathrm{P}_{2} \mathrm{~S}_{2} \mathrm{Sn}$ (606.98): C, 15.8; H, 3.0. Found: C, 15.7; H, 3.6\%. IR (Nujol/cm $\left.{ }^{-1}\right): \nu=531$ (s) (Sn-F). ${ }^{1} \mathrm{H}$ $\operatorname{NMR}\left(\mathrm{CD}_{2} \mathrm{Cl}_{2}, 298 \mathrm{~K}\right): \delta=1.88\left(\mathrm{~d},{ }^{2} J_{\mathrm{PH}}=12 \mathrm{~Hz}, \mathrm{CH}_{3}\right) \cdot{ }^{19} \mathrm{~F}\left\{{ }^{1} \mathrm{H}\right\}$ NMR ( $\mathrm{CD}_{2} \mathrm{Cl}_{2}, 298 \mathrm{~K}$ ): $\delta=-78.0$ (s, [6F], OTf), -140 (br s, [2F], $\mathrm{Sn}-\mathrm{F})$; $\left(\mathrm{CD}_{2} \mathrm{Cl}_{2}, 183 \mathrm{~K}\right): \delta=-77.8,-78.4\left(\mathrm{OTf}^{-}\right),-142.7\left(\mathrm{t},{ }^{2} J_{\mathrm{P}-}\right.$ $\left.=107 \mathrm{~Hz},{ }^{1} J_{119} \mathrm{SnF}=3393 \mathrm{~Hz},{ }^{1} J_{1_{11} \mathrm{SnF}}=3232, \mathrm{Sn}-\mathrm{F}\right) .{ }^{31} \mathrm{P}\left\{{ }^{1} \mathrm{H}\right\} \mathrm{NMR}$ $\left(\mathrm{CH}_{2} \mathrm{Cl}_{2}, 298 \mathrm{~K}\right): \delta=7.3(\mathrm{br}) ;\left(\mathrm{CH}_{2} \mathrm{Cl}_{2}, 183 \mathrm{~K}\right): \delta=10.2\left(\mathrm{t},{ }^{2} J_{\mathrm{PF}}\right.$ $\left.107 \mathrm{~Hz} ;{ }^{1} J_{119} \mathrm{SnP}=3654 \mathrm{~Hz} ;{ }^{1} J_{117} \mathrm{SnP}=3484\right) .{ }^{119} \mathrm{Sn} \mathrm{NMR}\left(\mathrm{CH}_{2} \mathrm{Cl}_{2}\right.$, $183 \mathrm{~K}): \delta=-609\left(\mathrm{tt},{ }^{1} J_{119 \mathrm{SnP}}=3654 \mathrm{~Hz},{ }^{1} J_{119} \mathrm{SnF}=3393 \mathrm{~Hz}\right)$.

\section{$\left[\mathrm{SnF}\left(\mathrm{PMe}_{3}\right)_{2}(\mathrm{OTf})_{3}\right]$}

To a solution of $\left[\mathrm{SnF}_{4}\left(\mathrm{PMe}_{3}\right)_{2}\right](0.100 \mathrm{~g}, 0.288 \mathrm{mmol})$ in $\mathrm{CH}_{2} \mathrm{Cl}_{2}$ $(2 \mathrm{~mL})$ a solution of TMSOTf $(0.192 \mathrm{~g}, 0.864 \mathrm{mmol})$ in $\mathrm{CH}_{2} \mathrm{Cl}_{2}$ 
(2 $\mathrm{mL}$ ) was added dropwise to form a clear solution. The reaction was stirred for $10 \mathrm{~min}$, the volatiles were then removed in vacuo to leave a solid, which was washed with hexane $(3 \times$ $10 \mathrm{~mL}$ ) and dried in vacuo to form a white powder. Yield $0.137 \mathrm{~g}$ (64\%). Required for $\mathrm{C}_{9} \mathrm{H}_{18} \mathrm{~F}_{10} \mathrm{O}_{9} \mathrm{P}_{2} \mathrm{~S}_{3} \mathrm{Sn}$ (737.07): C, 14.7; H, 2.6. Found: C, 14.3; H, 3.7\%. IR (Nujol $\left./ \mathrm{cm}^{-1}\right): \nu=$ 510m (Sn-F), 1158m, 1164m (-OSO $\left.{ }_{2}\right), 1191 \mathrm{~m}, 1202 \mathrm{~m}, 1237 \mathrm{~m}$, $1243 \mathrm{~m}\left(\mathrm{CF}_{3}\right) .{ }^{1} \mathrm{H} \mathrm{NMR}\left(\mathrm{CD}_{2} \mathrm{Cl}_{2}, 298 \mathrm{~K}\right): \delta=2.0\left(\mathrm{~d},{ }^{2} J_{\mathrm{PH}}=13 \mathrm{~Hz}\right.$, $\left.\mathrm{CH}_{3}\right) .{ }^{19} \mathrm{~F}\left\{{ }^{1} \mathrm{H}\right\} \operatorname{NMR}\left(\mathrm{CD}_{2} \mathrm{Cl}_{2}, 298 \mathrm{~K}\right): \delta=-77.6$ (s, [9F], OTf), $-132.9\left(\mathrm{t},{ }^{2} J_{\mathrm{PF}}=88 \mathrm{~Hz},{ }^{1} J_{119 \mathrm{SnF}}=3557 \mathrm{~Hz},{ }^{1} J_{117 \mathrm{SnF}}=3396 \mathrm{~Hz}\right)$, [F], (Sn-F). ${ }^{31} \mathrm{P}\left\{{ }^{1} \mathrm{H}\right\}$ NMR $\left(\mathrm{CD}_{2} \mathrm{Cl}_{2}, 298 \mathrm{~K}\right): \delta=18.3\left(\mathrm{~d},{ }^{2} J_{\mathrm{PF}}=88\right.$ $\left.\mathrm{Hz},{ }^{1} J_{119} \mathrm{SnP}=3740 \mathrm{~Hz} ;{ }^{1} J_{117} \mathrm{SnP}=3568 \mathrm{~Hz}\right) \cdot{ }^{119} \mathrm{Sn} \mathrm{NMR}\left(\mathrm{CH}_{2} \mathrm{Cl}_{2}\right.$, $183 \mathrm{~K}): \delta=-617(\mathrm{~m})$.

\section{$\left[\mathrm{SnF}_{4}\left(\mathbf{P}^{\mathrm{i}} \mathbf{P r}_{3}\right)_{2}\right]$}

To a suspension of $\left[\mathrm{SnF}_{4}(\mathrm{MeCN})_{2}\right](0.648 \mathrm{~g}, 2.34 \mathrm{mmol})$ in $\mathrm{CH}_{2} \mathrm{Cl}_{2}(5 \mathrm{~mL})$ a solution of $\mathrm{P}^{\mathrm{i}} \mathrm{Pr}_{3}(0.750 \mathrm{~g}, 4.68 \mathrm{mmol})$ in $\mathrm{CH}_{2} \mathrm{Cl}_{2}$ was added to form a slightly cloudy solution. The reaction was stirred for $1 \mathrm{~h}$, then the solution was filtered and the filtrate was concentrated in vacuo to yield a white solid, which was washed with hexane $(3 \times 10 \mathrm{~mL})$ and dried in vacuo. Yield: $0.862 \mathrm{~g}$ (71\%). $\mathrm{C}_{18} \mathrm{H}_{42} \mathrm{~F}_{4} \mathrm{P}_{2} \mathrm{Sn} \cdot 3 / 4 \mathrm{CH}_{2} \mathrm{Cl}_{2}$ (578.84): C, 38.9; $\mathrm{H}$, 7.6. Found: C, $38.8 \mathrm{H}, 8.1 \%$. IR (Nujol $\left./ \mathrm{cm}^{-1}\right): \nu=535 \mathrm{~s}(\mathrm{Sn}-\mathrm{F})$ ${ }^{1} \mathrm{H}$ NMR $\left(\mathrm{CD}_{2} \mathrm{Cl}_{2}, 298 \mathrm{~K}\right): \delta=1.41\left(\mathrm{dd},{ }^{3} J_{\mathrm{PH}}=15 \mathrm{~Hz},{ }^{3} J_{\mathrm{HH}}=7\right.$ $\mathrm{Hz},\left[18 \mathrm{H}\right.$ ], $\mathrm{CH}_{3}$ ), 2.55 (septet of d, ${ }^{3} J_{\mathrm{HH}}=7 \mathrm{~Hz},{ }^{2} J_{\mathrm{PH}}=1 \mathrm{~Hz}$, $[3 \mathrm{H}], \mathrm{CH}),{ }^{19} \mathrm{~F}\left\{{ }^{1} \mathrm{H}\right\} \mathrm{NMR}\left(\mathrm{CD}_{2} \mathrm{Cl}_{2}, 298 \mathrm{~K}\right): \delta=-99.1\left(\mathrm{t},{ }^{2} J_{\mathrm{PF}}=124\right.$ $\left.\mathrm{Hz},{ }^{1} J_{119} \mathrm{SnF}=2477 \mathrm{~Hz},{ }^{1} J_{{ }^{117} \mathrm{SnF}}=2363 \mathrm{~Hz}\right),{ }^{31} \mathrm{P}\left\{{ }^{1} \mathrm{H}\right\} \mathrm{NMR}$ $\left(\mathrm{CD}_{2} \mathrm{Cl}_{2}, 298 \mathrm{~K}\right): \delta=30.6$ (quint, ${ }^{2} J_{\mathrm{PF}}=124,{ }^{1} J_{119 \mathrm{SnP}}=2920 \mathrm{~Hz}$, $\left.{ }^{1} J_{117 \mathrm{SnP}}=2792 \mathrm{~Hz}\right) \cdot{ }^{119} \mathrm{Sn} \mathrm{NMR}\left(\mathrm{CH}_{2} \mathrm{Cl}_{2}, 183 \mathrm{~K}\right): \delta=-622.0$ (quin of t, ${ }^{1} J_{119} \mathrm{SnF}=2920 \mathrm{~Hz},{ }^{1} J_{117} \mathrm{SnP}=2477 \mathrm{~Hz}$ ).

\section{$\left[\mathrm{SnF}_{3}\left(\mathrm{P}^{\mathrm{i}} \mathrm{Pr}_{3}\right)_{2}(\mathrm{OTf})\right]$}

To a solution of $\left[\mathrm{SnF}_{4}\left(\mathrm{P}^{\mathrm{i}} \mathrm{Pr}_{3}\right)_{2}\right](0.100 \mathrm{~g}, 0.194 \mathrm{mmol})$ in $\mathrm{CH}_{2} \mathrm{Cl}_{2}$ $(2 \mathrm{~mL})$ a solution of TMSOTf $(0.043 \mathrm{~g}, 0.194 \mathrm{mmol})$ was added dropwise to form a clear solution. The reaction was stirred for $1 \mathrm{~h}$, volatiles were then removed in vacuo to yield a white solid, which was washed with hexane $(3 \times 10 \mathrm{~mL})$ and dried in vacuo. Yield: $0.095 \mathrm{~g}$ (76\%). Required for $\mathrm{C}_{19} \mathrm{H}_{42} \mathrm{~F}_{6} \mathrm{O}_{3} \mathrm{P}_{2} \mathrm{~S} \cdot 1 / 2 \mathrm{CH}_{2} \mathrm{Cl}_{2}$ (718.68): C, 32.6; H, 6.0. Found: C, 32.1; H, 6.7\%. IR (Nujol/ $\left.\mathrm{cm}^{-1}\right): \nu=540 \mathrm{~m}, 558 \mathrm{~m}, 571 \mathrm{~m}(\mathrm{Sn}-\mathrm{F}) .1150 \mathrm{~m}\left(-\mathrm{OSO}_{2}\right) 1223$, $1260\left(\mathrm{CF}_{3}\right) .{ }^{1} \mathrm{H}$ NMR $\left(\mathrm{CD}_{2} \mathrm{Cl}_{2}, 298 \mathrm{~K}\right): \delta=1.45\left(\mathrm{dd},{ }^{3} J_{\mathrm{PH}}=15 \mathrm{~Hz}\right.$, $\left.{ }^{3} J_{\mathrm{HH}}=7 \mathrm{~Hz},[18 \mathrm{H}], \mathrm{CH}_{3}\right), 2.77$ (septet of d, ${ }^{3} J_{\mathrm{HH}}=7 \mathrm{~Hz},{ }^{2} J_{\mathrm{PH}}=2$ $\mathrm{Hz}, \mathrm{CH}),{ }^{19} \mathrm{~F}\left\{{ }^{1} \mathrm{H}\right\} \mathrm{NMR}\left(\mathrm{CD}_{2} \mathrm{Cl}_{2}, 298 \mathrm{~K}\right): \delta=-73.5\left(\mathrm{br} \mathrm{s},{ }^{1} J_{\mathrm{SnF}}=\right.$ $3518 \mathrm{~Hz}$ ), -78.5 (s, OTf), -116.9 (br s, $\left.{ }^{1} J_{\mathrm{SnF}}=3244 \mathrm{~Hz}\right) .{ }^{31} \mathrm{P}\left\{{ }^{1} \mathrm{H}\right\}$ $\operatorname{NMR}\left(\mathrm{CH}_{2} \mathrm{Cl}_{2}, 298 \mathrm{~K}\right): \delta=38.2\left(\mathrm{q},{ }^{2} J_{\mathrm{PF}}=121 \mathrm{~Hz},{ }^{1} J_{119} \mathrm{SnP}=2827\right.$ $\left.\mathrm{Hz},{ }^{1} J_{117 \mathrm{SnP}}=2702 \mathrm{~Hz}\right),{ }^{119} \mathrm{Sn} \mathrm{NMR}\left(\mathrm{CH}_{2} \mathrm{Cl}_{2}, 183 \mathrm{~K}\right):-611.1(\mathrm{tdt}$, ${ }^{1} J_{119} \mathrm{SnF}=3558,3200 \mathrm{~Hz},{ }^{1} J_{119} \mathrm{SnP}=2856 \mathrm{~Hz}$.

\section{$\left[\mathrm{SnF}_{2}\left(\mathbf{P}^{\mathrm{i}} \mathrm{Pr}_{3}\right)_{2}(\mathrm{OTf})_{2}\right]$}

To a solution of $\left[\mathrm{SnF}_{4}\left(\mathrm{P}^{\mathrm{i}} \mathrm{Pr}_{3}\right)_{2}\right](0.200 \mathrm{~g}, 0.388 \mathrm{mmol})$ in $\mathrm{CH}_{2} \mathrm{Cl}_{2}$ $(2 \mathrm{~mL})$ a solution of TMSOTf $(0.173 \mathrm{~g}, 0.778 \mathrm{mmol})$ was added dropwise to form a clear solution. The reaction was stirred for $1 \mathrm{~h}$, volatiles were removed in vacuo to yield a white solid which was washed with hexane $(3 \times 10 \mathrm{~mL})$ and dried in vacuo. Yield: $0.231 \mathrm{~g}$ (77\%). Required for $\mathrm{C}_{20} \mathrm{H}_{42} \mathrm{~F}_{8} \mathrm{O}_{6} \mathrm{P}_{2} \mathrm{~S}_{2} \mathrm{Sn} \cdot \mathrm{CH}_{2} \mathrm{Cl}_{2}$ (860.20): C, 29.3; H, 5.2. Found: C, 29.1; H, 5.8\%. IR (Nujol/ $\left.\mathrm{cm}^{-1}\right): \nu=519 \mathrm{w}, 533 \mathrm{w}(\mathrm{Sn}-\mathrm{F}), 1150\left(\mathrm{OSO}_{2}\right), 1234 \mathrm{w}, 1261 \mathrm{w}$ $\left(\mathrm{CF}_{3}\right) .{ }^{1} \mathrm{H}$ NMR $\left(\mathrm{CD}_{2} \mathrm{Cl}_{2}, 298 \mathrm{~K}\right): 1.49\left(\mathrm{dd},{ }^{3} J_{\mathrm{PH}}=16 \mathrm{~Hz}[8 \mathrm{H}]\right.$, $\mathrm{CH}_{3}$ ), 3.05 (septet of d, ${ }^{3} J_{\mathrm{HH}}=8 \mathrm{~Hz},{ }^{2} J_{\mathrm{PH}}=3 \mathrm{~Hz},[3 \mathrm{H}], \mathrm{CH}$ ). ${ }^{19} \mathrm{~F}$ $\left\{{ }^{1} \mathrm{H}\right\} \mathrm{NMR}\left(\mathrm{CD}_{2} \mathrm{Cl}_{2}, 298 \mathrm{~K}\right):-56.9\left(\mathrm{br},{ }^{1} J_{\mathrm{SnF}}=4082 \mathrm{~Hz}\right),-77.9$ (s, OTf). ${ }^{31} \mathrm{P}\left\{{ }^{1} \mathrm{H}\right\} \mathrm{NMR}\left(\mathrm{CH}_{2} \mathrm{Cl}_{2}, 298 \mathrm{~K}\right): 49.4\left(\mathrm{t},{ }^{2} J_{\mathrm{PF}}=117 \mathrm{~Hz}\right.$,

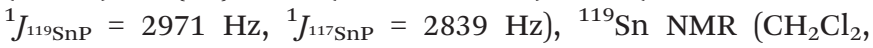
$183 \mathrm{~K}):-630\left(\mathrm{tt},{ }^{1} J_{119 \mathrm{SnF}}=4013,{ }^{1} J^{119 \mathrm{SnP}}=2941\right)$.

\section{$\left[\mathrm{SnF}_{4}(\right.$ triphos $\left.)\right]$}

To a suspension of $\left[\mathrm{SnF}_{4}(\mathrm{MeCN})_{2}\right](0.200 \mathrm{~g}, 0.722 \mathrm{mmol})$ in $\mathrm{CH}_{2} \mathrm{Cl}_{2}(2 \mathrm{~mL})$, triphos $(0.451 \mathrm{~g}, 0.722 \mathrm{mmol})$ was added as a solid and the resulting solution was stirred for $2 \mathrm{~h}$ to yield a cloudy solution, which was then filtered. Volatiles were removed from the filtrate in vacuo to yield a white solid, which was washed with hexane $(3 \times 10 \mathrm{~mL})$ and dried in vacuo to yield a white solid. Crystals suitable for single crystal X-ray diffraction were grown by layering a $\mathrm{CH}_{2} \mathrm{Cl}_{2}$ solution of the complex with hexane. Yield: $0.326 \mathrm{~g}$ (55\%). Required for $\mathrm{C}_{41} \mathrm{H}_{39} \mathrm{~F}_{4} \mathrm{P}_{3} \mathrm{Sn} \cdot 1 / 2 \mathrm{CH}_{2} \mathrm{Cl}_{2}$ (861.78): C, 57.8; H, 4.7. Found: C, $58.2 \mathrm{H}, 4.8 \%$. IR (Nujol $/ \mathrm{cm}^{-1}$ ): $\nu=516 \mathrm{~m}, 552 \mathrm{~m}, 564 \mathrm{~m}$ (Sn-F). ${ }^{1} \mathrm{H}$ NMR $\left(\mathrm{CD}_{2} \mathrm{Cl}_{2}, 298 \mathrm{~K}\right): \delta=0.87\left(\mathrm{~s},[3 \mathrm{H}], \mathrm{CH}_{3}\right), 2.28(\mathrm{~s},[2 \mathrm{H}])$, 2.91-2.95 (m, [2H]), 3.11 (m, [2H]), 7.1-8.1 (m, [30H], Ar-H). ${ }^{19} \mathrm{~F}\left\{{ }^{1} \mathrm{H}\right\} \operatorname{NMR}\left(\mathrm{CD}_{2} \mathrm{Cl}_{2}, 298 \mathrm{~K}\right): \delta=-144.2\left(\mathrm{tt},{ }^{2} J_{\mathrm{PF}}=98 \mathrm{~Hz},{ }^{2} J_{\mathrm{FF}}=\right.$ 45, [2F]), -109.7 (m, [2F]). ${ }^{31} \mathrm{P}\left\{{ }^{1} \mathrm{H}\right\}$ NMR $\left(\mathrm{CD}_{2} \mathrm{Cl}_{2}, 298 \mathrm{~K}\right): \delta=$ $-6.9\left(\mathrm{dtd},{ }^{2} J_{\mathrm{PF}}=121,98,51,[2 \mathrm{P}]\right),-27.1(\mathrm{~s},[\mathrm{P}]) .{ }^{119} \mathrm{Sn} \mathrm{NMR}$ $\left(\mathrm{CH}_{2} \mathrm{Cl}_{2}, 183 \mathrm{~K}\right): \delta=-681.4(\mathrm{~m})$.

\section{$\left[\mathrm{SnF}_{4}\left(\mathrm{dmso}_{2}\right]\right.$}

$\left[\mathrm{SnF}_{4}(\mathrm{MeCN})_{2}\right](0.20 \mathrm{~g}, 0.72 \mathrm{mmol})$ was suspended in excess dmso and stirred for $5 \mathrm{~min}$. The solvent was removed, and the white powder was dried in vacuo. Yield $0.120 \mathrm{~g}, 47 \%$. Required for $\mathrm{C}_{4} \mathrm{H}_{12} \mathrm{~F}_{4} \mathrm{O}_{2} \mathrm{~S}_{2} \mathrm{Sn}$ (351.0): C, 13.7; H, 3.5\%. Found C, 13.1; H, 3.5\%. IR ( Nujol $\left./ \mathrm{cm}^{-1}\right): \nu=936 \mathrm{~s}, 908 \mathrm{sh}(\mathrm{SO})$, 573vs, 552sh, 522m (SnF). ${ }^{1} \mathrm{H}$ NMR $\left(\mathrm{CD}_{3} \mathrm{NO}_{2}, 298 \mathrm{~K}\right): \delta=$ $3.1(\mathrm{~s}), 3.0(\mathrm{~s}) .{ }^{19} \mathrm{~F}\left\{{ }^{1} \mathrm{H}\right\} \mathrm{NMR}\left(\mathrm{CD}_{3} \mathrm{NO}_{2}, 298 \mathrm{~K}\right): \delta=-161.6(\mathrm{t}$, $\left.{ }^{1} J_{119} \mathrm{SnF}=2030,{ }^{2} J_{\mathrm{FF}}=52 \mathrm{~Hz}\right),-149.3\left(\mathrm{t},{ }^{1} J_{119 \mathrm{SnF}}=2020,{ }^{2} J_{\mathrm{FF}}=\right.$ $52 \mathrm{~Hz}),-149.0\left(\mathrm{~s},{ }^{1} J_{119 \mathrm{SnF}}=2466 \mathrm{~Hz}\right) .{ }^{119} \mathrm{Sn} \mathrm{NMR}\left(\mathrm{CH}_{3} \mathrm{NO}_{2}\right.$, $253 \mathrm{~K}): \delta=-778.4$.

\section{$\left[\mathrm{SnF}_{3}\left(\mathrm{dmso}_{3}\right][\mathrm{OTf}]\right.$}

$\left[\mathrm{SnF}_{4}(\mathrm{dmso})_{2}\right](0.15 \mathrm{~g}, 0.43 \mathrm{mmol})$ was suspended in $\mathrm{CH}_{2} \mathrm{Cl}_{2}$ $(10 \mathrm{~mL})$. To this TMSOTf $(0.035 \mathrm{~g}, 0.43 \mathrm{mmol})$ in $\mathrm{CH}_{2} \mathrm{Cl}_{2}$ $(5 \mathrm{~mL})$ was added and the solution stirred for $2 \mathrm{~h}$. Dmso $(0.04 \mathrm{~g}, 0.43 \mathrm{mmol})$ in $\mathrm{MeCN}(5 \mathrm{~mL})$ was then added and the solution was again stirred for $2 \mathrm{~h}$. The solvent and volatiles were removed and dried in vacuo, which yielded a colourless, gel-like solid which was recrystalised from $\mathrm{MeCN} / \mathrm{Et}_{2} \mathrm{O}$ to a colourless powder. Yield $0.11 \mathrm{~g}, 46 \%$. Required for $\mathrm{C}_{7} \mathrm{H}_{18} \mathrm{~F}_{6} \mathrm{O}_{6} \mathrm{~S}_{4} \mathrm{Sn}$ (559.2): C, 15.0; H, 3.2. Found C, 14.9; H, 3.2\%. IR (Nujol $\left./ \mathrm{cm}^{-1}\right): \nu=912 \mathrm{vbr}(\mathrm{SO}), 574 \mathrm{br}, 519 \mathrm{br}(\mathrm{SnF}) .{ }^{1} \mathrm{H}$ NMR $\left(\mathrm{CD}_{3} \mathrm{NO}_{2}, 298 \mathrm{~K}\right): \delta=3.11(\mathrm{~s}), 3.10(\mathrm{~s}), 3.08(\mathrm{~s}) .{ }^{19} \mathrm{~F}\left\{{ }^{1} \mathrm{H}\right\} \mathrm{NMR}$ $\left(\mathrm{CD}_{3} \mathrm{NO}_{2}, 298 \mathrm{~K}\right): \delta=-155.2\left(\mathrm{~s},{ }^{1} J_{119} \mathrm{SnF}=2063 \mathrm{~Hz}\right),-153.7(\mathrm{t}$, $\left.{ }^{1} J_{117} \mathrm{SnF}=1852,{ }^{1} J_{119} \mathrm{SnF}=2181 \mathrm{~Hz}\right),-145.8\left(\mathrm{~d},{ }^{1} J^{117} \mathrm{SnF}=1847\right.$, $\left.{ }^{1} J_{119 \mathrm{SnF}}=2063 \mathrm{~Hz},{ }^{2} J_{\mathrm{FF}}=59 \mathrm{~Hz}\right),-79.5\left(\mathrm{~s}\right.$, OTf). ${ }^{119} \mathrm{Sn} \mathrm{NMR}$ $\left(\mathrm{CH}_{3} \mathrm{NO}_{2}, 253 \mathrm{~K}\right): \delta=-744,-735$. 


\section{$\left[\mathrm{SnF}_{4}\left(\mathrm{pyNO}_{2}\right]\right.$}

$\left[\mathrm{SnF}_{4}(\mathrm{MeCN})_{2}\right](0.15 \mathrm{~g}, 0.54 \mathrm{mmol})$ was dissolved in $\mathrm{CH}_{2} \mathrm{Cl}_{2}$ and pyNO (0.10 gm, $1.08 \mathrm{mmol})$ was added. The solution was stirred for $2 \mathrm{~h}$. The white powder was filtered, washed in hexane $(3 \times 5 \mathrm{~mL})$ and dried in vacuo. Yield $0.125 \mathrm{~g}, 60 \%$. Required for $\mathrm{C}_{10} \mathrm{H}_{10} \mathrm{~F}_{4} \mathrm{~N}_{2} \mathrm{O}_{2} \mathrm{Sn}$ (384.9): C, 31.2; H, 2.6; N, 7.3. Found: C, 31.4; H, 2.6; N, 7.1\%. IR $\left(\mathrm{Nujol} / \mathrm{cm}^{-1}\right): \nu=1202 \mathrm{~m}$ (NO), 573br (Sn-F). ${ }^{1} \mathrm{H}$ NMR $\left(\mathrm{CD}_{3} \mathrm{NO}_{2}, 298 \mathrm{~K}\right): \delta=8.7(\mathrm{~m}$, $[2 \mathrm{H}]), 8.2(\mathrm{~m},[1 \mathrm{H}]), 7.9(\mathrm{~m},[2 \mathrm{H}]) .{ }^{19} \mathrm{~F}\left\{{ }^{1} \mathrm{H}\right\} \mathrm{NMR}\left(\mathrm{CD}_{3} \mathrm{NO}_{2}\right.$, $298 \mathrm{~K}): \delta=-168.7\left(\mathrm{t},{ }^{1} J_{119 \mathrm{SnF}}=2030,{ }^{2} J_{\mathrm{FF}}=51 \mathrm{~Hz}\right),-166.5(\mathrm{t}$, $\left.{ }^{1} J_{119 \mathrm{SnF}}=2081,{ }^{2} J_{\mathrm{FF}}=51 \mathrm{~Hz}\right),-164.2\left(\mathrm{~s},{ }^{1} J_{{ }^{119} \mathrm{SnF}}=1951\right) \cdot{ }^{119} \mathrm{Sn}$ $\mathrm{NMR}\left(\mathrm{CH}_{3} \mathrm{NO}_{2}, 253 \mathrm{~K}\right): \delta=-778.5$.

\section{$\left[\mathrm{SnF}_{3}\left(\mathrm{pyNO}_{3}\right][\mathrm{OTf}]\right.$}

$\left[\mathrm{SnF}_{4}(\mathrm{pyNO})_{2}\right](0.30 \mathrm{~g}, 0.78 \mathrm{mmol})$ was suspended in $\mathrm{CH}_{2} \mathrm{Cl}_{2}$. To this TMSOTf $(0.17 \mathrm{~g}, 0.78 \mathrm{mmol})$ was added in MeCN $(5 \mathrm{~mL})$. The solution was stirred for $2 \mathrm{~h}$. PyNO $(0.074 \mathrm{~g}$, $0.78 \mathrm{mmol}$ ) was added and the solution was stirred for a further $2 \mathrm{~h}$. The solvent was then removed in vacuo which yielded a colourless, gel-like solid which was recrystallised from $\mathrm{MeCN} / \mathrm{Et}_{2} \mathrm{O}$ to give a white powder. Yield: $0.22 \mathrm{~g}, 46 \%$. Required for $\mathrm{C}_{16} \mathrm{H}_{15} \mathrm{~F}_{6} \mathrm{~N}_{3} \mathrm{O}_{6} \mathrm{Sn} \cdot 1 / 2 \mathrm{CH}_{2} \mathrm{Cl}_{2}$ (652.54): C, 30.4; $\mathrm{H}$, 2.5; N, 6.4. Found: C, 30.3; H, 2.9; N, 6.6\%. IR (Nujol $\left./ \mathrm{cm}^{-1}\right): \nu$ $=1225 \mathrm{~m}(\mathrm{NO}), 574 \mathrm{br}, \mathrm{s}, 517 \mathrm{~m}(\mathrm{Sn}-\mathrm{F}) .{ }^{1} \mathrm{H}$ NMR $\left(\mathrm{CD}_{3} \mathrm{CN}, 298 \mathrm{~K}\right)$ : $\delta=8.8(\mathrm{~m},[2 \mathrm{H}]), 8.2(\mathrm{~m},[1 \mathrm{H}]), 7.9(\mathrm{~m},[2 \mathrm{H}]) .{ }^{19} \mathrm{~F}\left\{{ }^{1} \mathrm{H}\right\} \mathrm{NMR}$ $\left(\mathrm{CD}_{3} \mathrm{CN}, 298 \mathrm{~K}\right): \delta=-172.4\left(\mathrm{~s},{ }^{1} J^{119} \mathrm{SnF}=2159 \mathrm{~Hz}\right),-171.6(\mathrm{t}$, $\left.{ }^{1} J_{119 \mathrm{SnF}}=2268 \mathrm{~Hz},{ }^{2} J_{\mathrm{FF}}=59 \mathrm{~Hz}\right),-169.9\left(\mathrm{~d},{ }^{1} J_{119} \mathrm{SnF}=2244 \mathrm{~Hz}\right.$, ${ }^{2} J_{\mathrm{FF}}=59 \mathrm{~Hz}$ ), -78.7 (s, OTf). ${ }^{119} \mathrm{Sn} \mathrm{NMR}\left(\mathrm{CH}_{3} \mathrm{NO}_{2}, 253 \mathrm{~K}\right): \delta$ $\sim-770$ (multiplets for the two isomers are almost coincident).

\section{$\left[\mathrm{SnF}_{4}(\mathrm{DMF})_{2}\right]$}

$\left[\mathrm{SnF}_{4}(\mathrm{MeCN})_{2}\right](0.20 \mathrm{~g}, 0.72 \mathrm{mmol})$ was added to an excess of DMF $(5 \mathrm{~mL})$. The suspension was stirred for $2 \mathrm{~h}$. The white precipitate was filtered, washed in hexane $(3 \times 3 \mathrm{~mL})$ and dried in vacuo. Yield $0.195 \mathrm{~g}, 80 \%$. Required for $\mathrm{SnF}_{4} \mathrm{C}_{6} \mathrm{H}_{14} \mathrm{~N}_{2} \mathrm{O}_{2} \cdot \mathrm{CH}_{2} \mathrm{Cl}_{2}$ (425.8): C, 19.7; H, 3.8; N, 6.6. Found C, 19.5; H, 3.8; N, 7.4\%. IR (Nujol $\left./ \mathrm{cm}^{-1}\right): \nu=1669$ (CO), $585 \mathrm{~s}$ $(\mathrm{Sn}-\mathrm{F}) .{ }^{1} \mathrm{H}$ NMR $\left(\mathrm{CD}_{3} \mathrm{NO}_{2}, 298 \mathrm{~K}\right): \delta=8.16(\mathrm{~s}), 7.91(\mathrm{~s})(\mathrm{H}), 3.31$ (s), $3.28(\mathrm{~s}), 3.15(\mathrm{~s}), 3.12(\mathrm{~s}) \mathrm{CH}_{3} \cdot{ }^{19} \mathrm{~F}\left\{{ }^{1} \mathrm{H}\right\}$ NMR $\left(\mathrm{CD}_{3} \mathrm{NO}_{2}\right.$, $298 \mathrm{~K}): \delta=-169.5\left(\mathrm{t},{ }^{1} J_{119} \mathrm{SnF}=2015 \mathrm{~Hz},{ }^{2} J_{\mathrm{FF}}=50 \mathrm{~Hz}\right),-161.9(\mathrm{t}$, $\left.{ }^{1} J_{119 \mathrm{SnF}}=1907 \mathrm{~Hz},{ }^{2} J_{\mathrm{FF}}=50 \mathrm{~Hz}\right),-161.8(\mathrm{~s}) .{ }^{119} \mathrm{Sn} \mathrm{NMR}$ $\left(\mathrm{CH}_{3} \mathrm{NO}_{2}, 253 \mathrm{~K}\right)$ : n.o.

\section{$\left[\mathrm{SnF}_{3}(\mathrm{DMF})_{3}\right][\mathrm{OTf}]$}

$\left[\mathrm{SnF}_{4}(\mathrm{DMF})_{2}\right](0.31 \mathrm{~g}, 0.91 \mathrm{mmol})$ was suspended in $\mathrm{CH}_{2} \mathrm{Cl}_{2}$. To this TMSOTf $(0.20 \mathrm{~g}, 0.91 \mathrm{mmol})$ was added in $\mathrm{CH}_{2} \mathrm{Cl}_{2}$. The solution was stirred for $2 \mathrm{~h}$. DMF $(0.067 \mathrm{~g}, 0.91 \mathrm{mmol})$ in MeCN was then added and the solution was stirred for $2 \mathrm{~h}$. Addition of $n$-hexane formed a viscous oil from which the solvent was decanted and the residue was washed with further hexane and dried in vacuo, leaving a colourless glassy solid. Yield $0.085 \mathrm{~g}, 17 \%$. Microanalytical data were not obtained due to the glassy nature of the product. IR (Nujol $\left./ \mathrm{cm}^{-1}\right): \nu=1666$ $(\mathrm{C}=\mathrm{O}), 582 \mathrm{~s}(\mathrm{Sn}-\mathrm{F}), 518 \mathrm{~s}(\mathrm{Sn}-\mathrm{F}) .{ }^{1} \mathrm{H} \mathrm{NMR}\left(\mathrm{CD}_{3} \mathrm{NO}_{2}, 298 \mathrm{~K}\right): \delta=$ 8.27 (br s), 8.21 (s), 3.34 (m), 3.18 (m). ${ }^{19} \mathrm{~F}\left\{{ }^{1} \mathrm{H}\right\}$ NMR $\left(\mathrm{CD}_{3} \mathrm{NO}_{2}\right.$,
$298 \mathrm{~K}): \delta=-169.2\left(\mathrm{t},{ }^{1} J_{119 \mathrm{SnF}}=2143 \mathrm{~Hz},{ }^{2} J_{\mathrm{FF}}=55 \mathrm{~Hz}\right),-168.8(\mathrm{~s}$, $\left.{ }^{1} J_{119} \mathrm{SnF}=2150 \mathrm{~Hz}\right),-164.0\left(\mathrm{~d},{ }^{1} J_{119} \mathrm{SnF}=1985 \mathrm{~Hz},{ }^{2} J_{\mathrm{FF}}=55 \mathrm{~Hz}\right)$, -79.5 (s, OTf). ${ }^{119} \mathrm{Sn} \mathrm{NMR}\left(\mathrm{CH}_{3} \mathrm{NO}_{2}, 253 \mathrm{~K}\right)$ : n.o.

\section{$\left[\mathrm{SnF}_{3}(\mathrm{py})_{3}\right][\mathrm{OTf}]$}

$\left[\mathrm{SnF}_{4}(\mathrm{py})_{2}\right](0.10 \mathrm{~g}, 0.28 \mathrm{mmol})$ was suspended in $\mathrm{CH}_{2} \mathrm{Cl}_{2}$ $(10 \mathrm{~mL})$. To this TMSOTf $(0.063 \mathrm{~g}, 0.28 \mathrm{mmol})$ was added in $\mathrm{CH}_{2} \mathrm{Cl}_{2}(5 \mathrm{~mL})$. The solution was stirred for $2 \mathrm{~h}$. Py $(0.022 \mathrm{~g}$, $0.28 \mathrm{mmol}$ ) was added and the solution was stirred for a further $2 \mathrm{~h}$. The solvent was then removed in vacuo which yielded a colourless, gel-like solid which was recrystallised from $\mathrm{MeCN} / \mathrm{Et}_{2} \mathrm{O}$ to a white powder. Although we were unable to obtain satisfactory microanalytical data for this complex, the spectroscopic data are in accord with the formulation above. IR ( Nujol $\left./ \mathrm{cm}^{-1}\right)$ : $\nu=568 \mathrm{br}, \mathrm{s}(\mathrm{Sn}-\mathrm{F}) .{ }^{1} \mathrm{H}$ NMR $\left(\mathrm{CD}_{3} \mathrm{NO}_{2}, 298 \mathrm{~K}\right): \delta=8.9(\mathrm{~m}), 8.8$ (m), $8.4(\mathrm{~m}), 8.3(\mathrm{~m}), 7.9(\mathrm{~m}), 7.8(\mathrm{~m}) .{ }^{19} \mathrm{~F}\left\{{ }^{1} \mathrm{H}\right\}$ NMR $\left(\mathrm{CD}_{3} \mathrm{NO}_{2}\right.$, $298 \mathrm{~K}): \delta=-165.8\left(\mathrm{~m},{ }^{1} J_{117} \mathrm{SnF}=1637 \mathrm{~Hz},{ }^{1} J_{119} \mathrm{SnF}=1717 \mathrm{~Hz},{ }^{2} J_{\mathrm{FF}}=\right.$ $40 \mathrm{~Hz}),-158.8\left(\mathrm{~d},{ }^{1} J_{117} \mathrm{SnF}=1658 \mathrm{~Hz},{ }^{1} J_{119} \mathrm{SnF}=1737 \mathrm{~Hz},{ }^{2} J_{\mathrm{FF}}=\right.$ $40 \mathrm{~Hz}),-157.2\left(\mathrm{~s},{ }^{1} J^{117} \mathrm{SnF}=1515 \mathrm{~Hz},{ }^{1} J^{119} \mathrm{SnF}=1588 \mathrm{~Hz}\right),-79.9$ (s, OTf). ${ }^{119} \mathrm{Sn} \mathrm{NMR}\left(\mathrm{CH}_{3} \mathrm{NO}_{2}, 253 \mathrm{~K}\right)$ : n.o.

\section{$\left[\mathrm{SnF}_{3}\left(\mathrm{OPPh}_{3}\right)_{3}\right][\mathrm{OTf}]$}

$\left[\mathrm{SnF}_{4}\left(\mathrm{OPPh}_{3}\right)_{2}\right](0.073 \mathrm{~g}, 0.097 \mathrm{mmol})$ was dissolved in $\mathrm{CH}_{2} \mathrm{Cl}_{2}$ $(10 \mathrm{~mL})$. To this TMSOTf $(0.20 \mathrm{~g}, 0.91 \mathrm{mmol})$ was added in $\mathrm{CH}_{2} \mathrm{Cl}_{2}(5 \mathrm{~mL})$. The solution was stirred for $2 \mathrm{~h}$. To this $\mathrm{OPPh}_{3}$ $(0.027 \mathrm{~g}, 0.097 \mathrm{mmol})$ then added and the solution was stirred for $2 \mathrm{~h}$. The solution was concentrated in vacuo and excess hexane was added, the solvent was removed, and the resulting white powder was dried in vacuo. Yield $0.070 \mathrm{~g}, 63 \%$. IR (Nujol/ $\left.\mathrm{cm}^{-1}\right): \nu=1145 \mathrm{sh}, 1059(\mathrm{P}=\mathrm{O}), 554 \mathrm{~m}, 537 \mathrm{~m}(\mathrm{Sn}-\mathrm{F}) .{ }^{1} \mathrm{H}$ NMR $\left(\mathrm{CD}_{2} \mathrm{Cl}_{2}, 298 \mathrm{~K}\right): \delta=7.9-7.3(\mathrm{~m}) .{ }^{19} \mathrm{~F}\left\{{ }^{1} \mathrm{H}\right\} \mathrm{NMR}\left(\mathrm{CD}_{2} \mathrm{Cl}_{2}, 298 \mathrm{~K}\right)$ : $\delta=-142.1(\mathrm{~s}),-141.2\left(\mathrm{t},{ }^{2} J_{\mathrm{FF}}=61 \mathrm{~Hz}\right),-134.5\left(\mathrm{~d},{ }^{2} J_{\mathrm{FF}}=61 \mathrm{~Hz}\right)$, -79.1 (s, OTf). ${ }^{31} \mathrm{P}\left\{{ }^{1} \mathrm{H}\right\}$ NMR $\left(\mathrm{CD}_{2} \mathrm{Cl}_{2}, 298 \mathrm{~K}\right): \delta=46.0$ (s), 43.9 (s), 41.7 (s). ${ }^{119} \mathrm{Sn} \mathrm{NMR}\left(\mathrm{CH}_{3} \mathrm{NO}_{2}, 253 \mathrm{~K}\right)$ : n.o.

\section{$\left[\mathrm{SnF}_{2}\left(\mathrm{OPPh}_{3}\right)_{4}\right][\mathrm{OTf}]_{2}$}

$\left[\mathrm{SnF}_{4}\left(\mathrm{OPPh}_{3}\right)_{2}\right](0.085 \mathrm{~g}, 0.11 \mathrm{mmol})$ was dissolved in $\mathrm{CH}_{2} \mathrm{Cl}_{2}$ $(5 \mathrm{~mL})$. To this TMSOTf $(0.050 \mathrm{~g}, 0.23 \mathrm{mmol})$ was added in $\mathrm{CH}_{2} \mathrm{Cl}_{2}(5 \mathrm{~mL})$. The suspension was stirred for $2 \mathrm{~h}$ and then $\mathrm{OPPh}_{3}(0.063 \mathrm{~g}, 0.23 \mathrm{mmol})$ was added and the solution was stirred for a further $2 \mathrm{~h}$. The solution was concentrated in vacuo and excess hexane was added, the solvent was decanted, and the resulting white powder was dried in vacuo. Yield $0.08 \mathrm{~g}, 47 \%$. Required for $\mathrm{C}_{74} \mathrm{H}_{60} \mathrm{~F}_{8} \mathrm{O}_{10} \mathrm{P}_{4} \mathrm{~S}_{2} \cdot \mathrm{CH}_{2} \mathrm{Cl}_{2}$ (1652.9): C, 54.5; H, 3.8. Found: C, 54.6; H, 3.8\%. IR (Nujol $\left./ \mathrm{cm}^{-1}\right): \nu=1150$ (sh), $1060(\mathrm{P}=\mathrm{O}), 550 \mathrm{~s}, 537 \mathrm{~s}, 517 \mathrm{~m}(\mathrm{Sn}-\mathrm{F}) .{ }^{1} \mathrm{H} \mathrm{NMR}\left(\mathrm{CD}_{3} \mathrm{NO}_{2}\right.$, $298 \mathrm{~K}): \delta=7.3-7.9(\mathrm{~m}) .{ }^{19} \mathrm{~F}\left\{{ }^{1} \mathrm{H}\right\}$ NMR $\left(\mathrm{CD}_{3} \mathrm{NO}_{2}, 298 \mathrm{~K}\right): \delta=$ $-123.1\left(\mathrm{~s},{ }^{1} J_{117} \mathrm{SnF}=1730,{ }^{1} J_{119} \mathrm{SnF}=1812 \mathrm{~Hz}\right),-122.4\left(\mathrm{~s},{ }^{1} J_{117} \mathrm{SnF}=\right.$ $\left.1977,{ }^{1} J_{119} \mathrm{SnF}=2069 \mathrm{~Hz}\right),-79.8$ (s, OTf). ${ }^{31} \mathrm{P}\left\{{ }^{1} \mathrm{H}\right\} \quad \mathrm{NMR}$ $\left(\mathrm{CD}_{3} \mathrm{NO}_{2}, 298 \mathrm{~K}\right): \delta=50.5\left(\mathrm{~s},{ }^{1} J_{\mathrm{SnP}}=78 \mathrm{~Hz}\right), 48.4\left(\mathrm{~s},{ }^{1} J_{\mathrm{SnP}}=78\right.$ $\mathrm{Hz}), 47.1\left(\mathrm{~s},{ }^{1} J_{\mathrm{SnP}}=96 \mathrm{~Hz}\right.$ ) average ${ }^{117} \mathrm{Sn} /{ }^{119} \mathrm{Sn}$; separate couplings were not resolved. ${ }^{119} \mathrm{Sn} \mathrm{NMR}\left(\mathrm{CH}_{3} \mathrm{NO}_{2}, 253 \mathrm{~K}\right)$ : n.o.

\section{X-Ray experimental}

Crystals of the phosphine complexes were grown by layering $\mathrm{CH}_{2} \mathrm{Cl}_{2}$ solutions with hexane, while those for the $\mathrm{N}$ - and 
O-donor complexes were obtained by slow evaporation from $\mathrm{MeNO}_{2}$ solutions in the glovebox. Data collections used a Rigaku AFC12 goniometer equipped with an enhanced sensitivity (HG) Saturn724+ detector mounted at the window of an FR-E+ SuperBright molybdenum $(\lambda=0.71073 \AA)$ rotating anode generator with VHF Varimax optics (70 $\mu \mathrm{m}$ focus) with the crystal held at $100 \mathrm{~K}$. Structure solution and refinement were performed using SHELX(S/L)97, SHELX-2013, or SHELX-2014/ $7.41 .^{21,22} \mathrm{H}$ atoms bonded to $\mathrm{C}$ were placed in calculated positions using the default $\mathrm{C}-\mathrm{H}$ distance and refined using a riding model. The structure of $\left[\mathrm{SnF}_{4}(\mathrm{py})_{2}\right]$ showed some twinning, which we were not able to fully resolve, hence the final structure quality if less good than for the other complexes reported. Details of the crystallographic parameters are given in Table $\mathrm{S} 1$ (ESI $\dagger$ ). CCDC reference numbers for the crystallographic information files in cif format are CCDC 2104490 ([Sn( $\left.\left.\left(\mathrm{PMe}_{3}\right)_{2}(\mathrm{OTf})_{2}\right]\right), 2104491 \quad\left(\mathrm{Sn}_{3} \mathrm{~F}_{5} \mathrm{OTf}\right), 2104492$ $\left(\left[\mathrm{SnF}_{3}\left(\mathrm{PMe}_{3}\right)_{2}(\mathrm{OTf})\right]\right), 2104493\left(\left[\mathrm{SnF}_{4}\left(\kappa^{2}\right.\right.\right.$-triphos $\left.\left.)\right]\right), \quad 2104976$ $\left.\left(\left[\mathrm{SnF}_{2}\left(\mathrm{OPPh}_{3}\right)_{4}\right)\right][\mathrm{OTf}]_{2}\right), 2106812\left(\left[\mathrm{SnF}_{4}(\mathrm{py})_{2}\right]\right.$ and 2104984 $\left(\left[\mathrm{SnF}_{4}\left(\mathrm{pyNO}_{2}\right]\right) \cdot \dagger\right.$

\section{Results and discussion}

The neutral tin(Iv) fluoride complex trans-[SnF $\left.4\left(\mathrm{PMe}_{3}\right)_{2}\right]$ was synthesised following the literature $\operatorname{method}^{7}$ and two new complexes $\left[\mathrm{SnF}_{4}\left(\mathrm{P}^{\mathrm{i}} \mathrm{Pr}_{3}\right)_{2}\right]$ and $\left[\mathrm{SnF}_{4}\left(\kappa^{2}\right.\right.$-triphos $\left.)\right]$ (triphos =

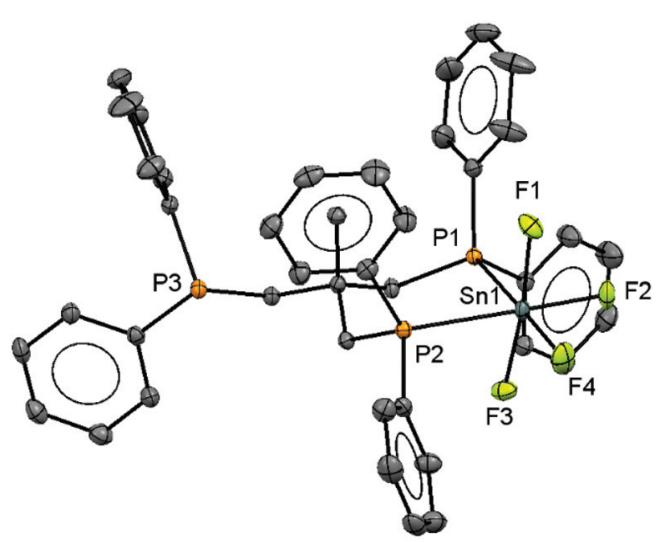

Fig. 1 Crystal structure of $\left[\mathrm{SnF}_{4}\left(\kappa^{2}\right.\right.$-triphos $\left.)\right]$ showing the atom labelling scheme. The ellipsoids are drawn at the $50 \%$ probability level and $\mathrm{H}$ atoms are omitted for clarity. Selected bond lengths $(\AA)$ and angles $\left({ }^{\circ}\right)$ are: $\mathrm{Sn} 1-\mathrm{P} 1=2.6302(5), \mathrm{Sn} 1-\mathrm{P} 2=2.6314(5) \mathrm{Sn} 1-\mathrm{F} 1=1.9670(12), \mathrm{Sn} 1-$ $F 2=1.9488(12), S n 1-F 3=1.9791(12), S n 1-F 4=1.9307(12), P 1-S n 1-P 2$ $=91.245(14), \mathrm{P} 1-\mathrm{Sn} 1-\mathrm{F} 4=172.46(5), \mathrm{P} 2-\mathrm{Sn} 1-\mathrm{F} 2=174.74(4), \mathrm{F} 1-\mathrm{Sn} 1-$ $F 3=173.44(5)$.
$\left.\mathrm{CH}_{3} \mathrm{C}\left(\mathrm{CH}_{2} \mathrm{PPh}_{2}\right)_{3}\right)$ were made similarly. The presence of a quintet in the ${ }^{31} \mathrm{P}\left\{{ }^{1} \mathrm{H}\right\}$ and a triplet in the ${ }^{19} \mathrm{~F}\left\{{ }^{1} \mathrm{H}\right\}$ NMR spectrum of $\left[\mathrm{SnF}_{4}\left(\mathrm{P}^{\mathrm{i}} \mathrm{Pr}_{3}\right)_{2}\right]$ shows this exists only as the trans isomer as found for other tertiary phosphine complexes of $\mathrm{SnF}_{4}{ }^{7}{ }^{7}$ The reaction of $\left[\mathrm{SnF}_{4}(\mathrm{MeCN})_{2}\right]$ with triphos leads to the formation of the complex $\left[\mathrm{SnF}_{4}\left(\kappa^{2}\right.\right.$-triphos $\left.)\right]$. The ${ }^{31} \mathrm{P}\left\{{ }^{1} \mathrm{H}\right\}$ NMR spectrum of this complex at $298 \mathrm{~K}$ in $\mathrm{CH}_{2} \mathrm{Cl}_{2}$ solution is a doublet of triplets of doublets at $\delta=-6.9 \mathrm{ppm}$ and a singlet at $\delta=$ -27.1 ppm with integrals in a $2: 1$ ratio, consistent with a $\kappa^{2}$ coordinated triphos in solution, and with no evidence of other isomers present. The crystal structure of the complex (Fig. 1) confirms this, and there is no evidence that the free arm can spontaneously displace a fluoride from the metal centre, a result similar to that found in the heavier tin(Iv) halide complexes. ${ }^{8}$ The geometry at the tin is distorted octahedral and the $\mathrm{Sn}-\mathrm{F}$ and $\mathrm{Sn}-\mathrm{P}$ bond lengths are much as expected. ${ }^{8}$

\section{Fluoride abstraction from $\left[\mathrm{SnF}_{4}\left(\mathrm{PR}_{3}\right)_{2}\right]$ complexes}

Since neutral phosphine ligands do not displace fluoride from the tin centre directly, the reactions with the potential fluoride abstraction reagents ${ }^{9} \mathrm{Na}\left[\mathrm{BAr}^{\mathrm{F}}\right]$ and $\mathrm{Me}_{3} \mathrm{SiO}_{3} \mathrm{SCF}_{3}$ (TMSOTf) were explored. Some reaction was evident with $\mathrm{Na}\left[\mathrm{BAr}^{\mathrm{F}}\right]$, but did not go to completion ( $c f$. ref. 10) and hence studies were focussed on TMSOTf (Scheme 1). The reaction of trans$\left[\mathrm{SnF}_{4}\left(\mathrm{PMe}_{3}\right)_{2}\right]$ with one equivalent of TMSOTf in $\mathrm{CH}_{2} \mathrm{Cl}_{2}$ leads to the formation of $\left[\mathrm{SnF}_{3}\left(\mathrm{PMe}_{3}\right)_{2}(\mathrm{OTf})\right]$. The crystal structure of this complex (Fig. 2) shows that in the solid state the three fluorines are in a mer-arrangement with OTf trans-F and with mutually trans- $\mathrm{PMe}_{3}$ ligands. The same isomer is present in $\left[\mathrm{SnCl}_{3}\left(\mathrm{PMe}_{3}\right)_{2}(\mathrm{OTf})\right]{ }^{10}$ and comparison of the structures shows the $\mathrm{d}(\mathrm{Sn}-\mathrm{P})$ and $\mathrm{d}(\mathrm{Sn}-\mathrm{O})$ are only marginally shorter in the fluoride complex.

In general the triflate complexes are less robust than the $\left[\mathrm{SnF}_{4}\left(\mathrm{PR}_{3}\right)_{2}\right]$ and resonances of decomposition products, which include $\left[\mathrm{R}_{3} \mathrm{PF}\right]^{+}$, build up quite rapidly in solution, especially for the mono-fluoride species. In solution at $298 \mathrm{~K}$ the $\left[\mathrm{SnF}_{3}\left(\mathrm{PMe}_{3}\right)_{2}(\mathrm{OTf})\right]$ exhibits broad ${ }^{19} \mathrm{~F}\left\{{ }^{1} \mathrm{H}\right\}$ and ${ }^{31} \mathrm{P}\left\{{ }^{1} \mathrm{H}\right\}$ NMR spectra, probably due to some reversible OTf dissociation. However, cooling the solution to $183 \mathrm{~K}$ sharpened these resonances; in the ${ }^{31} \mathrm{P}\left\{{ }^{1} \mathrm{H}\right\}$ NMR spectrum there is a triplet of doublets and in the ${ }^{19} \mathrm{~F}\left\{{ }^{1} \mathrm{H}\right\}$ NMR spectrum a triplet of triplets and a triplet of doublets are seen in a $1: 2$ ratio (Table 1 and Fig. 3), as expected if the structure in Fig. 2 was retained in solution. Obtaining ${ }^{119} \mathrm{Sn}$ NMR spectra proved to be difficult from the triflate complexes, even at $183 \mathrm{~K}$, the full couplings were often unclear, although the chemical shifts were identified.

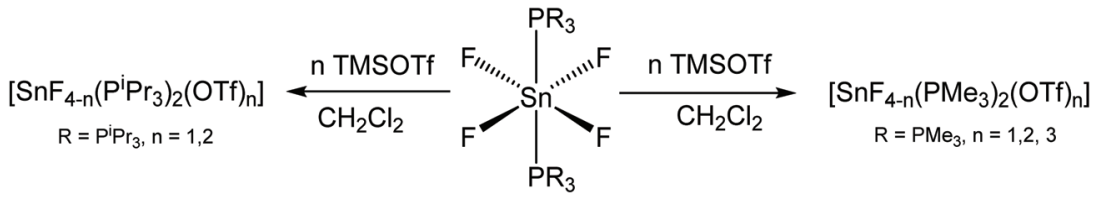




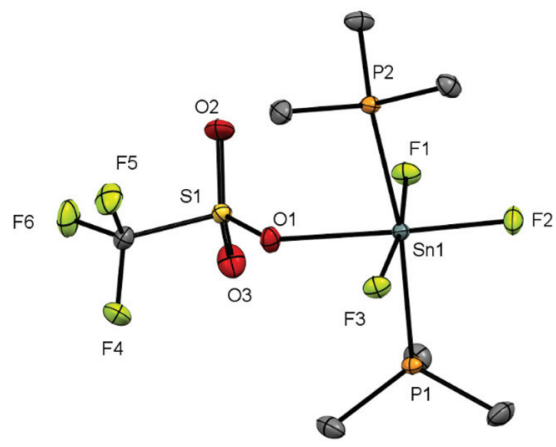

Fig. 2 Crystal structure of $\left[\mathrm{SnF}_{3}\left(\mathrm{PMe}_{3}\right)_{2}(\mathrm{OTf})\right]$ showing the atom labelling scheme. The ellipsoids are drawn at the $50 \%$ probability level and $\mathrm{H}$ atoms are omitted for clarity. Selected bond lengths $(\AA)$ and angles $\left(^{\circ}\right)$ are: Sn1-P1 = 2.5278(4), Sn1-P2 = 2.5405(4), Sn1-F1 = 1.9761(9), Sn1$\mathrm{F} 2=1.9595(10), \mathrm{Sn} 1-\mathrm{F} 3=1.9871(9), \mathrm{Sn} 1-\mathrm{O} 1=2.2445(12), \mathrm{P} 1-\mathrm{Sn} 1-\mathrm{P} 2=$ 171.615(13), F1-Sn1-F3 = 171.00(4), O1-Sn1-F3 = 176.66(4).

The reaction of trans-[ $\left.\mathrm{SnF}_{4}\left(\mathrm{PMe}_{3}\right)_{2}\right]$ with two equivalents of TMSOTf forms the complex $\left[\mathrm{SnF}_{2}\left(\mathrm{PMe}_{3}\right)_{2}(\mathrm{OTf})_{2}\right]$ as identified by the microanalysis. Here the low temperature ${ }^{19} \mathrm{~F}\left\{{ }^{1} \mathrm{H}\right\}$ and ${ }^{31} \mathrm{P}\left\{{ }^{1} \mathrm{H}\right\}$ NMR spectra are triplets which suggests that the complex has trans phosphines, with the fluorides either cis or trans. At $183 \mathrm{~K}$ both the ${ }^{19} \mathrm{~F}\left\{{ }^{1} \mathrm{H}\right\}$ and ${ }^{31} \mathrm{P}\left\{{ }^{1} \mathrm{H}\right\}$ NMR spectra (ESI S3.2 and S3.3†) show, in addition to resonances of $\left[\mathrm{SnF}_{2}\left(\mathrm{PMe}_{3}\right)_{2}(\mathrm{OTf})_{2}\right]$, some minor species which may be $\left[\mathrm{SnF}_{2}\left(\mathrm{PMe}_{3}\right)_{2}(\mathrm{OTf})_{2-n}\right][\mathrm{OTf}]_{n}$. Attempts to grow crystals for an $\mathrm{X}$-ray study to confirm the geometry of $\left[\mathrm{SnF}_{2}\left(\mathrm{PMe}_{3}\right)_{2}(\mathrm{OTf})_{2}\right]$ failed due to limited solution stability, and instead gave some crystals of the tin(II) complex, $\left[\mathrm{Sn}\left(\mathrm{PMe}_{3}\right)_{2}(\mathrm{OTf})_{2}\right]$ (see below). The reaction of trans-[ $\left[\mathrm{SnF}_{4}\left(\mathrm{PMe}_{3}\right)_{2}\right]$ with three equivalents of TMSOTf leads to the formation of the complex [SnF $\left.\left(\mathrm{PMe}_{3}\right)_{2}(\mathrm{OTf})_{3}\right]$. The ${ }^{19} \mathrm{~F}\left\{{ }^{1} \mathrm{H}\right\}$ and ${ }^{31} \mathrm{P}\left\{{ }^{1} \mathrm{H}\right\}$ spectra of this complex are sharp at room temperature; the ${ }^{31} \mathrm{P}\left\{{ }^{1} \mathrm{H}\right\}$ NMR spectrum shows a doublet resonance indicating equivalent phosphines (mutually trans), whilst the ${ }^{19} \mathrm{~F}\left\{{ }^{1} \mathrm{H}\right\}$ NMR spectrum has two resonances, a singlet corresponding to triflate and a triplet corresponding to the tin bound fluoride, in a 9:1 ratio. This complex is not stable in solution and over a number of hours these resonances decrease in intensity and new resonances consistent with the formation of $\left[\mathrm{PMe}_{3} \mathrm{~F}\right]^{+}$appear. ${ }^{23} \mathrm{~A}$ few crystals deposited from the solution were revealed by structure solution to be a phosphine free tin(II) polymer, $\mathrm{Sn}_{3} \mathrm{~F}_{5}$ (OTf) (see below).

The sequential abstraction of fluoride from $\left[\mathrm{SnF}_{4}\left(\mathrm{PMe}_{3}\right)_{2}\right]$ leads to a high frequency shift in the phosphorus resonance and an increase in ${ }^{1} J_{119} \mathrm{SnP}$ and ${ }^{1} J_{119} \mathrm{SnF}$, which is consistent with an increase in charge at the tin centre. There is a decrease in ${ }^{2} J_{\mathrm{PF}}$ as the series is traversed. The triflate has a trans influence on the fluorides, best demonstrated in the monotriflate complex where both the ${ }^{2} J_{\mathrm{PF}}$ and ${ }^{1} J_{119 \mathrm{SnF}}$ couplings are smaller for the trans fluorine than for the cis. The ${ }^{119} \mathrm{Sn}$ NMR spectra are expected to be complex, and in some the couplings are not all clearly resolved even at $183 \mathrm{~K}$ (Table 1 and ESI†े).

In an attempt to generate cations, the reaction of TMSOTf with trans-[$\left[\mathrm{SnF}_{4}\left(\mathrm{P}^{\mathrm{i}} \mathrm{Pr}_{3}\right)_{2}\right]$ was explored. The $\mathrm{P}^{\mathrm{i}} \mathrm{Pr}_{3}$ has a Tolman cone angle $e^{24}$ of $160^{\circ}$ which compares with $118^{\circ}$ in $\mathrm{PMe}_{3}$. Whilst it was possible to sequentially remove one or two fluorines from the tin, the resulting complexes had very limited stability and attempts to obtain X-ray crystallographic data were unsuccessful. However, based upon the microanalysis and multinuclear NMR data they were formulated as $\left[\mathrm{SnF}_{3}\left(\mathrm{P}^{\mathrm{i}} \mathrm{Pr}_{3}\right)_{2}(\mathrm{OTf})\right]$ and $\left[\mathrm{SnF}_{2}\left(\mathrm{P}^{\mathrm{i}} \mathrm{Pr}_{3}\right)_{2}(\mathrm{OTf})_{2}\right]$, with the NMR data suggesting the triflates are most likely coordinated to the tin in solution at low temperature, like the trimethylphosphine complexes, although dissociated at room temperature. The reaction of $\left[\mathrm{SnF}_{4}\left(\mathrm{P}^{\mathrm{i}} \mathrm{Pr}_{3}\right)_{2}\right]$ with one equivalent of TMSOTf produces the complex $\left[\mathrm{SnF}_{3}\left(\mathrm{P}^{\mathrm{i}} \mathrm{Pr}_{3}\right)_{2}(\mathrm{OTf})\right]$. The room temperature ${ }^{31} \mathrm{P}\left\{{ }^{1} \mathrm{H}\right\}$ NMR spectrum of which is a quartet, indicating the three Sn-bound $\mathrm{F}^{-}$groups are equivalent, whilst the ${ }^{19} \mathrm{~F}\left\{{ }^{1} \mathrm{H}\right\}$ NMR spectrum has broad resonances flanked by tin satellites (as well as a triflate resonance), which sharpen as the temperature is lowered to $183 \mathrm{~K}$. The room temperature data support the tentative assignment of the tin species as a trans-trigonal bipyramid, although cation-OTf interactions most likely occur at $183 \mathrm{~K}$ and in the solid state.

The reaction of $\left[\mathrm{SnF}_{4}\left(\mathrm{P}^{\mathrm{i}} \mathrm{Pr}_{3}\right)_{2}\right]$ with two equivalents of TMSOTf leads to the formation of $\left[\operatorname{SnF}_{2}\left(\mathrm{P}^{\mathrm{i}} \mathrm{Pr}_{3}\right)_{2}(\mathrm{OTf})_{2}\right]$. The ${ }^{31} \mathrm{P}$ $\left\{{ }^{1} \mathrm{H}\right\}$ NMR spectrum of this complex is a triplet, whilst the ${ }^{19} \mathrm{~F}$ $\left\{{ }^{1} \mathrm{H}\right\}$ spectrum is a broad resonance at $\delta=-57.0$, which is flanked by tin satellites. It is likely that weak interactions with the triflate anions may be present in solution, and the solution decomposes quickly. Curiously, the reaction of TMSOTf with $\left[\mathrm{SnCl}_{4}\left(\mathrm{ER}_{3}\right)_{2}\right]\left(\mathrm{E}=\mathrm{P}\right.$ or As) only generated $\left[\mathrm{SnCl}_{3}\left(\mathrm{ER}_{3}\right)_{2} \mathrm{OTf}\right]$; further halides were not removed even with the addition of excess TMSOTf. ${ }^{10}$

As described above, decomposition of some of the triflate complexes in solution produced crystals of tin(II) compounds. From $\left[\mathrm{SnF}_{2}\left(\mathrm{PMe}_{3}\right)_{2}(\mathrm{OTf})_{2}\right]$ one decomposition product was [Sn $\left.\left(\mathrm{PMe}_{3}\right)_{2}(\mathrm{OTf})_{2}\right]$ (Fig. 4), which has a four-coordinate primary coordination sphere around the Sn(II) centre, based upon a trigonal bipyramid with equatorial phosphines, axial triflates and a vacant equatorial vertex. There are weak long-range contacts between neighbouring molecules through a Sn-triflate interaction $(3.044 \AA)$ to yield a 1D polymer (ESI Fig. S8†). The Sn-P bond distances in this species are $c a$. $0.2 \AA$ longer and the Sn-OTf $c a$. 0.3 $\AA$ shorter than those in the Sn(rv) complex, $\left[\mathrm{SnF}_{3}\left(\mathrm{PMe}_{3}\right)_{2}(\mathrm{OTf})\right]$ (above).

One product from the decomposition of $\left[\mathrm{SnF}\left(\mathrm{PMe}_{3}\right)_{2}(\mathrm{OTf})_{3}\right]$ in solution is the $\mathrm{Sn}$ (II) phosphine-free compound, $\mathrm{Sn}_{3} \mathrm{~F}_{5}(\mathrm{OTf})$, which has an extended 2D sheet structure formed mainly through Sn-F bridges (see ESI. Fig. S9†).

\section{Fluoro-tin(Iv) cations with hard $\mathrm{N}$ - and $\mathrm{O}$-donor ligands}

The work described above shows that fluoride can be abstracted sequentially from tetrafluorotin(Iv) phosphine complexes, to form tri-, di- and mono-fluoride complexes in which OTf coordination replaces the fluorides removed. However, these complexes mostly have limited stability, and attempts to introduce further phosphine into the coordination sphere, for example by reacting TMSOTf with $\left[\mathrm{SnF}_{4}\left(\kappa^{2}\right.\right.$-triphos)] (bearing a pendant $-\mathrm{PPh}_{2}$ function) does not lead to formation of the 


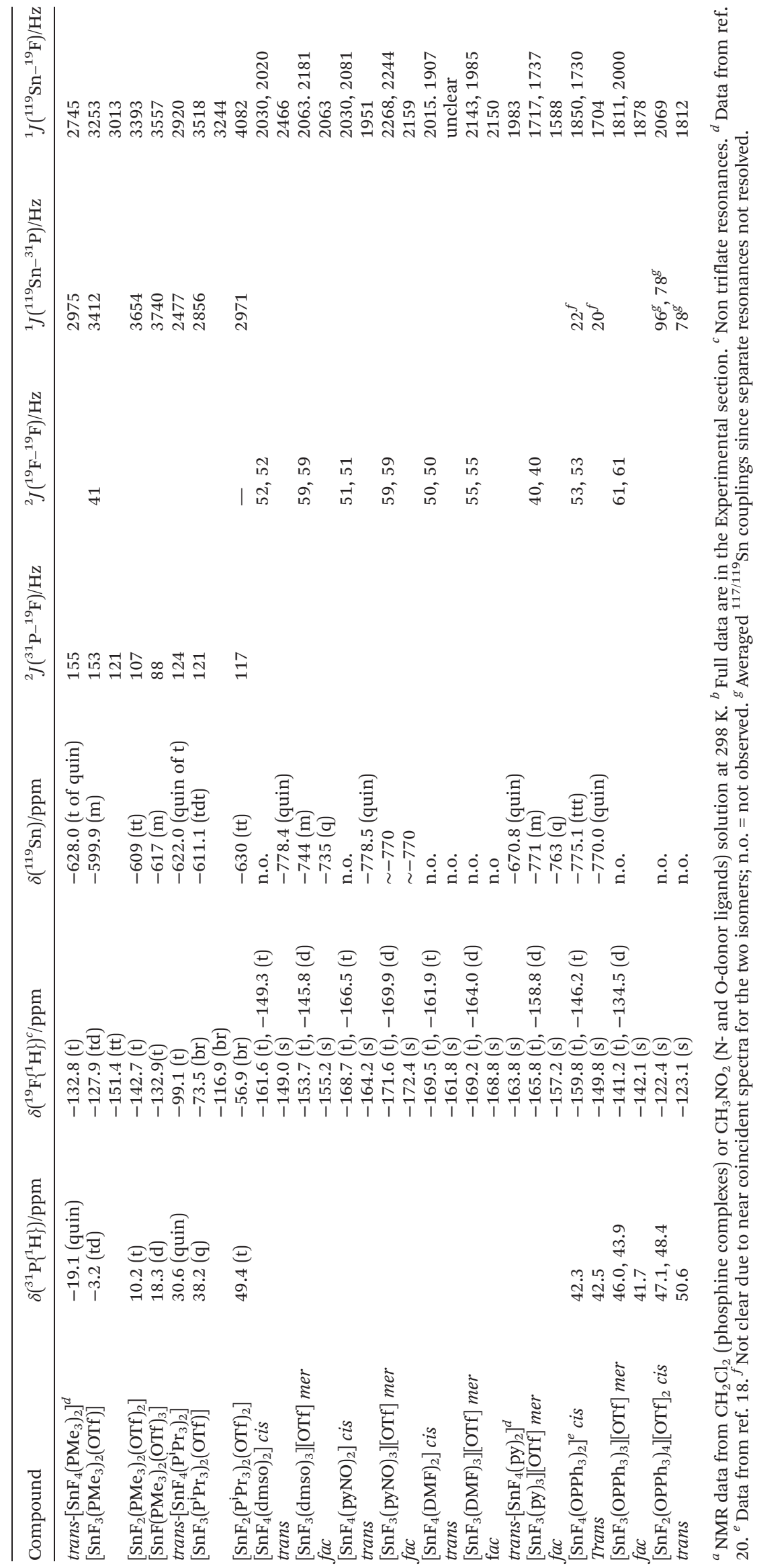




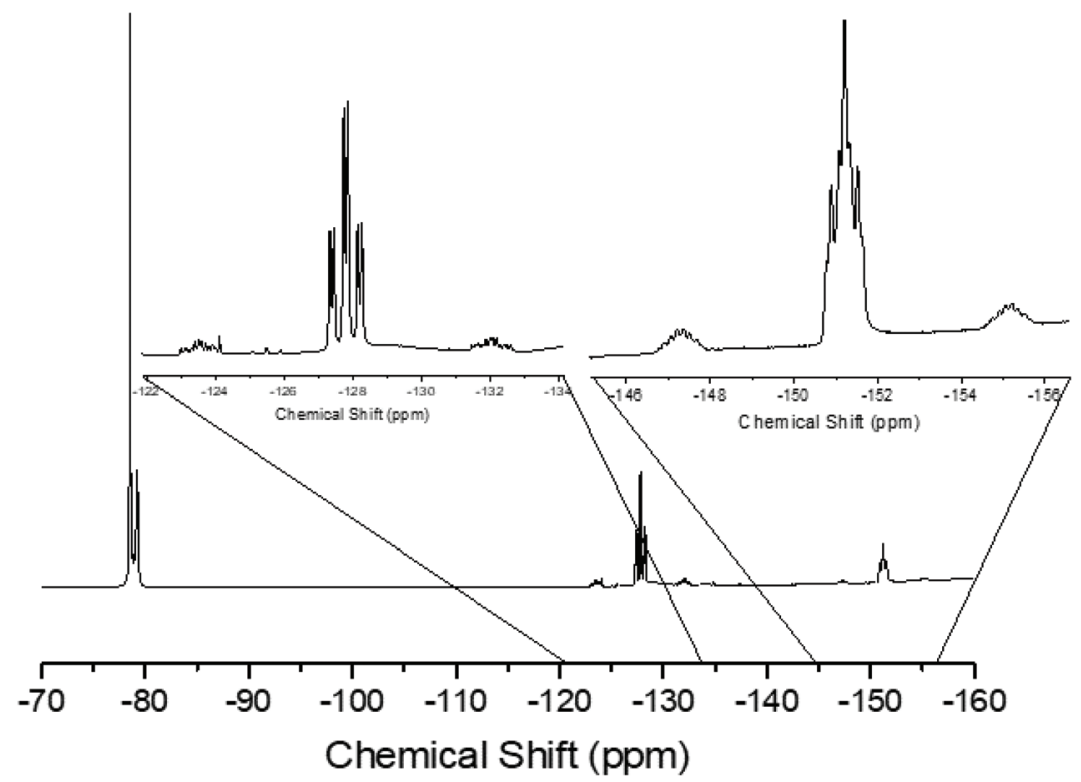

Fig. 3 The ${ }^{19} \mathrm{~F}\left\{{ }^{1} \mathrm{H}\right\} \mathrm{NMR}$ spectrum of $\left[\mathrm{SnF}_{3}\left(\mathrm{PMe}_{3}\right)_{2}(\mathrm{OTf})\right]\left(\mathrm{CD}_{2} \mathrm{Cl}_{2}, 183 \mathrm{~K}\right)$.

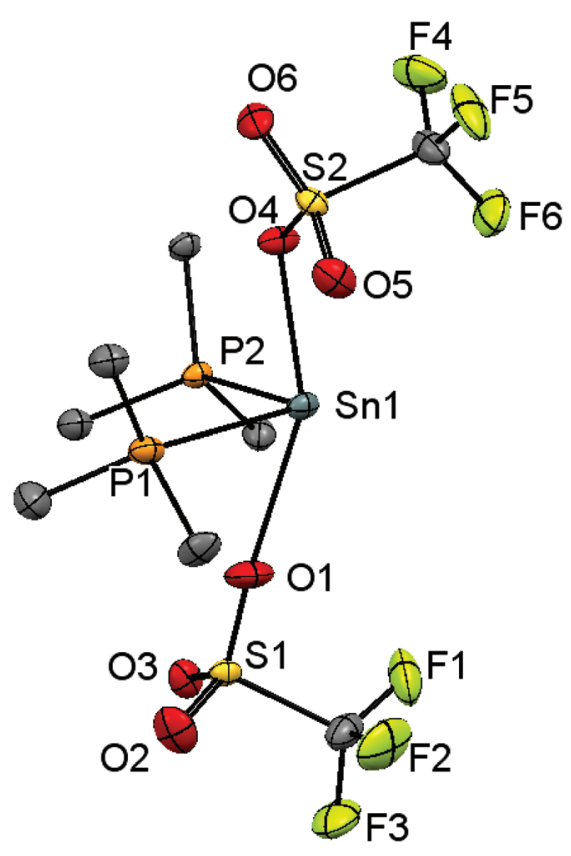

Fig. 4 Crystal structure of $\left[\mathrm{Sn}\left(\mathrm{PMe}_{3}\right)_{2}(\mathrm{OTf})_{2}\right]$ showing the atom labelling scheme. The ellipsoids are drawn at the $50 \%$ probability level and $\mathrm{H}$ atoms are omitted for clarity. Selected bond lengths $(\AA)$ and angles $\left({ }^{\circ}\right)$ are: Sn1-P1 = 2.7796(10), Sn1-P2 = 2.6610(8), Sn1-O1 = 2.523(3), Sn1$\mathrm{O} 4=2.342(3), \mathrm{P} 1-\mathrm{Sn} 1-\mathrm{P} 2=96.27(3), \mathrm{O} 1-\mathrm{Sn} 1-\mathrm{O} 4=154.39(10)$.

intended $\left[\mathrm{SnF}_{3}\left(\mathrm{\kappa}^{3}\right.\right.$-triphos $\left.)\right][\mathrm{OTf}]$. Similarly, adding $\mathrm{PMe}_{3}$ to $\left[\mathrm{SnF}_{3}\left(\mathrm{PMe}_{3}\right)_{2}(\mathrm{OTf})\right]$ did not lead to clean substitution of the OTf. Hence, in this section we explore fluoride abstraction from tetrafluorotin(Iv) complexes containing hard, neutral nitrogen and oxygen donor ligands in the presence of further neutral ligand. The neutral complexes $\left[\operatorname{SnF}_{4}(\mathrm{~L})_{2}\right](\mathrm{L}=\mathrm{dmso}$, pyNO, $\mathrm{OPPh}_{3}$, py, DMF) have been reported in the earlier literature, ${ }^{8,13,14,19}$ but most with limited data. The key spectroscopic data are given in Table 1 and the Experimental section. The structures of trans- $\left[\mathrm{SnF}_{4}\left(\mathrm{OPR}_{3}\right)_{2}\right](\mathrm{R}=\mathrm{Me}, \mathrm{Ph})^{8,19}$ have been determined previously and those of trans-[ $\left.\mathrm{SnF}_{4}(\mathrm{pyNO})_{2}\right]$ and trans-[ $\left.\mathrm{SnF}_{4}(\mathrm{py})_{2}\right]$ are shown in Fig. 5 . The NMR spectra of

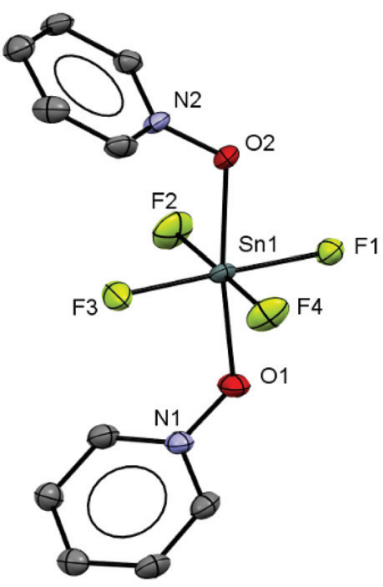

(a)

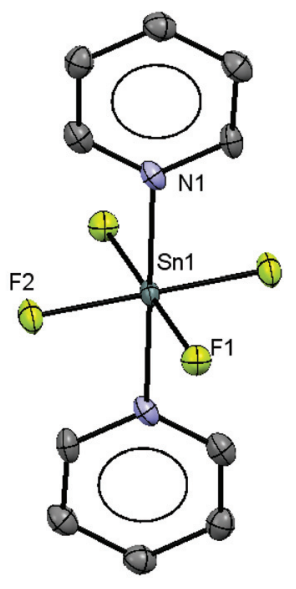

(b)
Fig. 5 Crystal structures of (a) $\left[\mathrm{SnF}_{4}(\mathrm{pyNO})_{2}\right] \cdot \mathrm{CH}_{2} \mathrm{Cl}_{2}$ and (b) the centrosymmetric $\left[\mathrm{SnF}_{4}(\mathrm{py})_{2}\right]$ showing the atom labelling schemes. The ellipsoids are drawn at the $50 \%$ probability level and $\mathrm{H}$ atoms and lattice $\mathrm{CH}_{2} \mathrm{Cl}_{2}$ are omitted for clarity. There are two crystallographically independent $\left[\mathrm{SnF}_{4}(\text { pyNO) })_{2}\right] \cdot \mathrm{CH}_{2} \mathrm{Cl}_{2}$ moieties in asymmetric unit and only one is shown. Selected bond lengths $(\AA \AA)$ and angles $\left(^{\circ}\right)$ are: (a) Sn1-F1 = 1.945(3), Sn1-F2 = 1.945(4), Sn1-F3 = 1.943(4), Sn1-F4 = 1.953(3), Sn1$\mathrm{O} 1=2.081(3), \mathrm{Sn} 1-\mathrm{O} 2=2.081(3), \mathrm{N} 1-\mathrm{O} 1=1.364(5), \mathrm{N} 1-\mathrm{O} 2=1.359(5)$, O1-Sn1-O2 = 172.29(15), F1-Sn1-F3 = 177.59(16), F2-Sn1-F4 = 177.20 (16); (b) Sn1-F1 = 1.954(7), Sn1-F2 = 1.956(7), Sn1-N1 = 2.175(10), F1$\mathrm{Sn} 1-\mathrm{F} 2=90.1(3), \mathrm{F} 1-\mathrm{Sn} 1-\mathrm{N} 1=90.0(3), \mathrm{F} 2-\mathrm{Sn} 1-\mathrm{N} 1=90.0(3)$. 


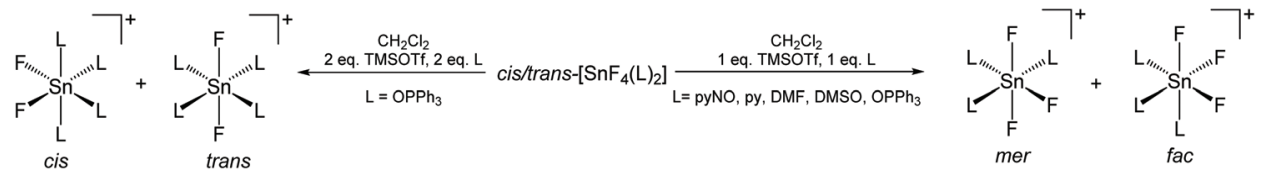

Scheme 2

most of the $\left[\mathrm{SnF}_{4}(\mathrm{~L})_{2}\right]$ complexes show both cis and trans isomers present in solution, with the relative amounts varying significantly with L (Table 1) (Scheme 2).

The complexes were mostly poorly soluble in $\mathrm{CH}_{2} \mathrm{Cl}_{2}$ and generally solution data were acquired from $\mathrm{CH}_{3} \mathrm{NO}_{2} / \mathrm{CD}_{3} \mathrm{NO}_{2}$ solutions, with stronger donor solvents being avoided since these may displace the neutral ligands from the tin. The high melting point of $\mathrm{MeNO}_{2}(244 \mathrm{~K})$ limited low temperature NMR studies. The isomers present are readily identified from the ${ }^{19} \mathrm{~F}\left\{{ }^{1} \mathrm{H}\right\}$ NMR spectra with the trans showing a sharp singlet resonance and the cis, two triplets, in both cases with accompanying ${ }^{117 / 119} \mathrm{Sn}$ satellites. The relative amounts of the two isomers are solvent dependent, although we have not explored this in any detail. The ${ }^{1} J_{\mathrm{SnF}}$ couplings are large and usually the separate couplings to the two tin isotopes were resolved, despite their similar values (the magnetogyric ratio ${ }^{119} \mathrm{Sn} /{ }^{117} \mathrm{Sn}$ is 1.046) (Table 1). A typical example is shown in Fig. 6.
The reactions of the $\left[\operatorname{SnF}_{4}(\mathrm{~L})_{2}\right]$ complexes with one equivalent of TMSOTf, followed by addition of one equivalent of $\mathrm{L}$, afforded $\left[\mathrm{SnF}_{3}(\mathrm{~L})_{3}\right][\mathrm{OTf}]\left(\mathrm{L}=\mathrm{dmso}\right.$, py, pyNO, DMF, $\left.\mathrm{OPPh}_{3}\right)$. The ${ }^{19} \mathrm{~F}\left\{{ }^{1} \mathrm{H}\right\}$ NMR spectra show that in solution both the merand $f a c$-isomers are present, the former with doublet and triplet resonances due to ${ }^{2} J_{\mathrm{FF}}$, and the latter a singlet, again all with ${ }^{117 / 119} \mathrm{Sn}$ isotope satellites. A typical example is shown in Fig. 7.

Observing the ${ }^{119} \mathrm{Sn}$ spectra proved more challenging due to a combination of factors, including the presence of two isomers, the complex multiplet patterns which often overlapped, and the modest receptivity of ${ }^{119} \mathrm{Sn}\left(D_{\mathrm{c}}=26\right)$. Typically, the central lines of the multiplets were identified, allowing $\delta\left({ }^{119} \mathrm{Sn}\right)$ be determined, but the weaker outer lines of the multiplets were often less clear. Several complexes, including $\left[\mathrm{SnF}_{4}(\mathrm{DMF})_{2}\right], \quad\left[\mathrm{SnF}_{3}(\mathrm{DMF})_{3}\right][\mathrm{OTf}]$ and $\left[\mathrm{SnF}_{3}\left(\mathrm{OPPh}_{3}\right)_{3}\right][\mathrm{OTf}]$, failed to exhibit a ${ }^{119} \mathrm{Sn}$ resonance (in $\mathrm{CH}_{3} \mathrm{NO}_{2}$ at $253 \mathrm{~K}$ ), pre-

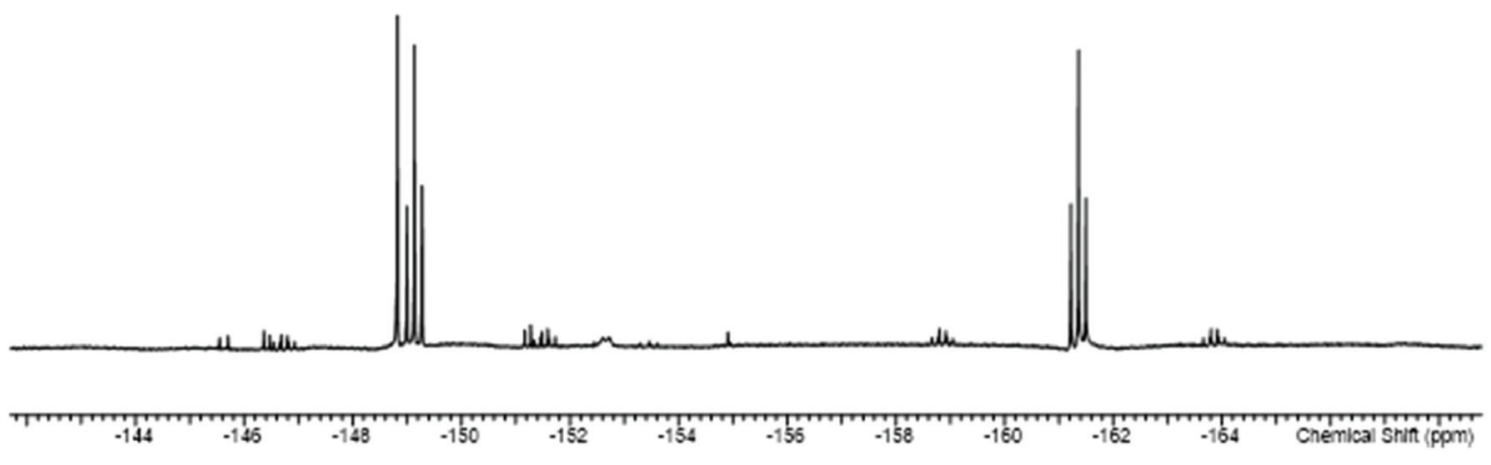

Fig. 6 The ${ }^{19} \mathrm{~F}\left\{{ }^{1} \mathrm{H}\right\}$ NMR spectrum of $\left[\mathrm{SnF}_{4}\left(\mathrm{dmso}_{2}\right](298 \mathrm{~K})\right.$ showing the resonances of the two isomers with ${ }^{117 / 119} \mathrm{Sn}$ satellites.

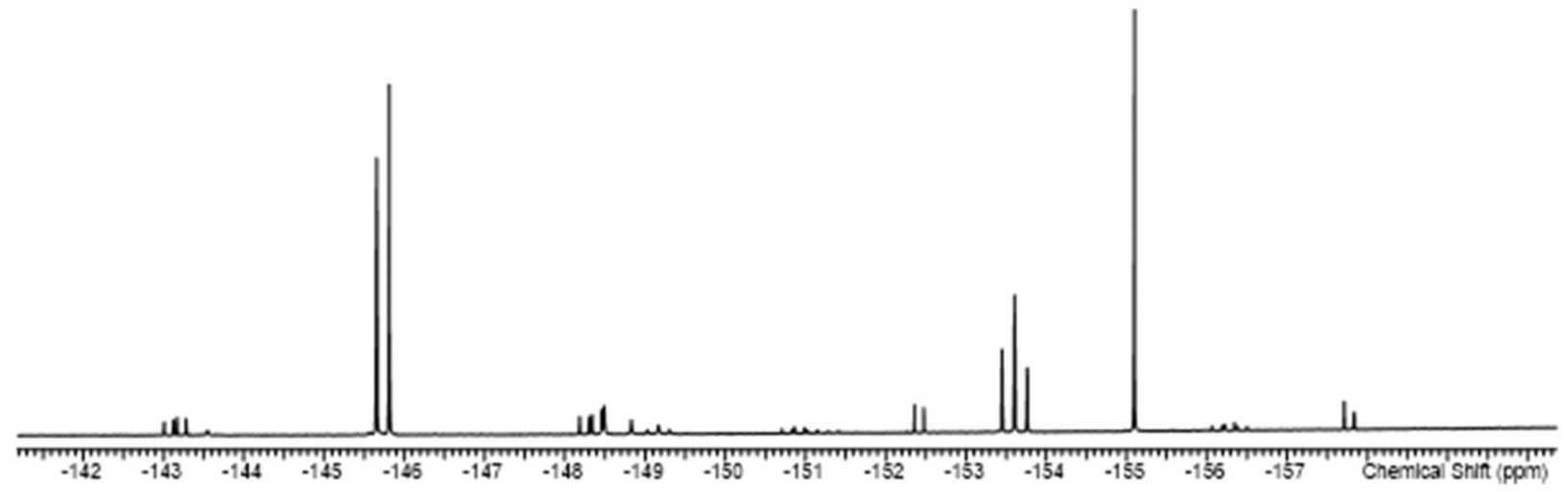

Fig. 7 The ${ }^{19} \mathrm{~F}\left\{{ }^{1} \mathrm{H}\right\}$ NMR spectrum of $\left[\mathrm{SnF}_{3}(\mathrm{dmso})_{3}\right][\mathrm{OTf}](298 \mathrm{~K})$ showing the resonances of the two isomers with ${ }^{117 / 119} \mathrm{Sn}$ satellites (the OTf resonance is not shown). 
sumably due to reversible neutral ligand dissociation on the tin NMR timescale. Similar behaviour has been reported for $\left[\mathrm{SnF}_{4}(\mathrm{MeCN})_{2}\right]^{8}$ Attempts to isolate pure samples of difluoro$\operatorname{tin}(\mathrm{Iv})$ cations $\left[\mathrm{SnF}_{2}(\mathrm{~L})_{4}\right][\mathrm{OTf}]_{2}$ from $\left[\operatorname{SnF}_{4}(\mathrm{~L})_{2}\right]$, with two equivalents of TMSOTf and excess L, proved more challenging. Typically, mixtures of $\left[\mathrm{SnF}_{2}(\mathrm{~L})_{4}\right][\mathrm{OTf}]_{2}$ and $\left[\operatorname{SnF}_{3}(\mathrm{~L})_{3}\right]$ OTf were identified in situ by the ${ }^{19} \mathrm{~F}\left\{{ }^{1} \mathrm{H}\right\}$ NMR spectra. It did prove possible to isolate an analytically pure sample of $\left[\mathrm{SnF}_{2}\left(\mathrm{OPPh}_{3}\right)_{4}\right][\mathrm{OTf}]_{2}$. The ${ }^{19} \mathrm{~F}\left\{{ }^{1} \mathrm{H}\right\}$ and ${ }^{31} \mathrm{P}\left\{{ }^{1} \mathrm{H}\right\}$ NMR spectra of this complex confirmed that both cis and trans isomers of the phosphine oxide complex were present in $\mathrm{CH}_{3} \mathrm{NO}_{2}$ solution, with the cis form the more abundant. However, we did not observe $\mathrm{a}^{119} \mathrm{Sn}$ NMR spectrum from this complex even at $253 \mathrm{~K}$ (the lower limit for $\mathrm{CH}_{3} \mathrm{NO}_{2}$ ). The X-ray crystal structure of $\left[\mathrm{SnF}_{2}\left(\mathrm{OPPh}_{3}\right)_{4}\right][\mathrm{OTf}]_{2}$ showed it to be of the trans isomer (Fig. 8).

The tin environment is very close to a regular octahedron and the $\mathrm{d}(\mathrm{Sn}-\mathrm{F})$ are rather shorter than in the neutral $\left[\mathrm{SnF}_{4}(\mathrm{~L})_{2}\right](\mathrm{L}=$ pyNO, py) complexes above, although the d(Sn$\mathrm{O}$ ) are not significantly different to those in the neutral parent complex trans $-\left[\mathrm{SnF}_{4}\left(\mathrm{OPPh}_{3}\right)_{2}\right]^{8}$

The $\left[\mathrm{SnF}_{3}(\mathrm{~L})_{3}\right][\mathrm{OTf}]$ complexes are less robust in solution than the neutral $\left[\mathrm{SnF}_{4}(\mathrm{~L})_{2}\right]$, and upon standing, resonances corresponding to varying amounts of decomposition/disproportionation products, including $\left[\mathrm{SnF}_{4}(\mathrm{~L})_{2}\right]$, were observed in some systems, especially for $\mathrm{L}=\mathrm{OPPh}_{3}$ and DMF. It is notable that the crystal used to determine the structure of $\left[\mathrm{SnF}_{4}(\mathrm{py})_{2}\right]$ (above) was grown from a sample of $\left[\mathrm{SnF}_{3}(\mathrm{py})_{3}\right][\mathrm{OTf}]$, and our inability to isolate pure $\left[\mathrm{SnF}_{2}(\mathrm{~L})_{4}\right][\mathrm{OTf}]_{2}$ with other $\mathrm{N}$ - or O-donor ligands suggest some ligand redistribution occurs in solution.

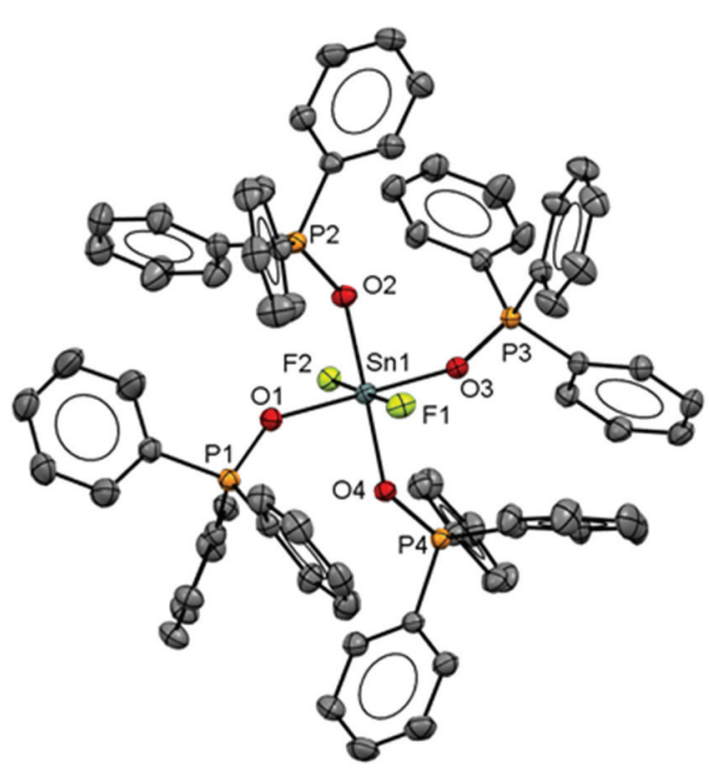

Fig. 8 Structure of the cation in $\left[\mathrm{SnF}_{2}\left(\mathrm{OPPh}_{3}\right)_{4}\right][\mathrm{OTf}]_{2}$ showing the atom labelling scheme. The ellipsoids are drawn at the $50 \%$ probability level and $\mathrm{H}$ atoms and OTf anions are omitted for clarity. Selected bond lengths $(\AA \AA)$ and angles $\left(^{\circ}\right)$ are: Sn1-F2 = 1.9314(16), Sn1-F1 = 1.9164(17), $\mathrm{Sn} 1-\mathrm{O} 3=2.040(2), \mathrm{Sn} 1-\mathrm{O} 4=2.0447(19), \mathrm{Sn} 1-\mathrm{O} 2=2.040(2), \mathrm{Sn} 1-\mathrm{O} 1$ $=2.057(2), \mathrm{F} 1-\mathrm{Sn} 1-\mathrm{F} 2=178.78(7), \mathrm{O} 3-\mathrm{Sn} 1-\mathrm{O} 1=176.24(8), \mathrm{O}-2 \mathrm{Sn} 1-$ O4 $=178.85(8)$.

\section{Conclusions}

A series of new triflate complexes of tin(rv) fluoride with neutral donor co-ligands have been synthesised. Variable temperature multinuclear NMR spectroscopic data show that soft phosphine ligands lead to neutral tri- and (unstable) di- and mono-fluoride complexes with two trans phosphines and one, two or three $\kappa^{1}$ OTf groups, respectively. The triflate is not displaced by added phosphine (even using the potentially tridentate triphos ligand). In contrast, harder $\mathrm{O}-$ and $\mathrm{N}$-donor ligands form the six-coordinate mer- and fac-[ $\left[\mathrm{SnF}_{3} \mathrm{~L}_{3}\right]^{+}\left(\mathrm{L}=\mathrm{dmso}, \mathrm{dmf}, \mathrm{OPPh}_{3}\right.$, pyNO and py) monocations, with further TMSOTf producing cisand trans-[ $\left[\mathrm{SnF}_{2}\left(\mathrm{OPPh}_{3}\right)_{4}\right]^{2+}$ dications, the latter being confirmed as the trans isomer in the solid state by X-ray analysis.

\section{Conflicts of interest}

The authors have no conflicts to declare.

\section{Acknowledgements}

We thank EPSRC for funding via the ADEPT Programme grant (EP/N035437/1), a studentship to R. P. K. (EP/N509747/1) and GE Healthcare and EPSRC for a CASE studentship to M. S. W. (EP/R513325/1).

\section{References}

1 (a) S. Aldridge and C. Jones, Chem. Soc. Rev., 2016, 45, 763 and references therein; (b) K. Chansaenpak, B. Vabre and F. P. Gabbal, Chem. Soc. Rev., 2016, 45, 954; (c) J. Burt, W. Levason and G. Reid, Coord. Chem. Rev., 2014, 260, 65; (d) C. I. Rat, C. Silvestru and H. J. Breunig, Coord. Chem. Rev., 2013, 257, 818; (e) T. J. Hadlington, M. Hermann, G. Frenking and C. Jones, J. Am. Chem. Soc., 2014, 136, 3028; (f) R. D. Rittinghaus, J. Tremmel, A. Růžička, C. Conrads, P. Albrecht, A. Hoffman, A. N. Ksiazkiewicz, A. Pich, R. Jambor and S. Herres-Pawlis, Chem. - Eur. J., 2020, 26, 212; $(g)$ C. H. de Groot, C. Gurnani, A. L. Hector, R. Huang, M. Jura, W. Levason and G. Reid, Chem. Mater., 2012, 24, 4442.

2 P. G. Harrison, in Comprehensive Coordination Chemistry, ed. G. Wilkinson, J. A. McCleverty and R. D. Gillard, Pergamon, Oxford, 1988, vol. 3, p. 183.

3 J. Parr, in Comprehensive Coordination Chemistry II, ed. J. A. McCleverty and T. J. Meyer, Elsevier, Oxford, 2004, vol. 3, p. 545 .

4 The Chemistry of Tin, ed. P. J. Smith, Chapman \& Hall, London, 1998.

5 P. G. Harrison, The Chemistry of Tin, Blackie, London, 1989.

6 G. R. Willey, T. J. Woodman, U. Somasundaram, D. R. Aris and W. Errington, J. Chem. Soc., Dalton Trans., 1998, 2575.

7 G. R. Willey, A. Jarvis, J. Palin and W. Errington, J. Chem. Soc., Dalton Trans., 1994, 255. 
8 M. F. Davis, M. Clarke, W. Levason, G. Reid and M. Webster, Eur. J. Inorg. Chem., 2006, 2773.

9 E. MacDonald, L. Doyle, S. S. Chitnis, U. WernerZwanziger, N. Burford and A. Decken, Chem. Commun., 2012, 48, 7922.

10 V. K. Greenacre, R. P. King, W. Levason and G. Reid, Dalton Trans., 2019, 48, 17097.

11 M. Bork and R. Hoppe, Z. Anorg. Allg. Chem., 1996, 622, 1557.

12 (a) S. L. Benjamin, W. Levason and G. Reid, Chem. Soc. Rev., 2013, 42, 1460; (b) W. Levason, F. M. Monzittu and G. Reid, Coord. Chem. Rev., 2019, 391, 90.

13 C. E. Michelson, D. S. Dyer and R. O. Ragsdale, J. Inorg. Nucl. Chem., 1970, 32, 833.

14 C. J. Wilkins and H. M. Haendler, J. Chem. Soc., 1965, 3174.

15 S. H. Hunter, V. M. Langford, G. A. Rodley and C. J. Wilkins, J. Chem. Soc. A, 1968, 305.
16 A. D. Adley, P. H. Bird, A. R. Fraser and M. Onyszchuk, Inorg. Chem., 1972, 11, 1402.

17 J. P. Clark, V. M. Langford and C. J. Wilkins, J. Chem. Soc. A, 1967, 792.

18 (a) D. Tudela and F. Rey, Z. Anorg. Allg. Chem., 1989, 575, 202; (b) D. Tudela and F. Patron, Inorg. Synth., 1997, 31, 92.

19 M. F. Davis, W. Levason, G. Reid and M. Webster, Polyhedron, 2006, 25, 930.

20 R. Suter, A. Swidan, C. L. B. Macdonald and N. Burford, Chem. Commun., 2018, 54, 4140.

21 (a) G. M. Sheldrick, Acta Crystallogr., Sect. C: Struct. Chem., 2015, 71, 3; (b) G. M. Sheldrick, Acta Crystallogr., Sect. A: Found. Crystallogr., 2008, 64, 112.

22 O. V. Dolomanov, L. J. Bourhis, R. J. Gildea, J. A. K. Howard and H. Puschmann, J. Appl. Crystallogr., 2009, 42, 339.

23 A. M. Forster and A. J. Downs, Polyhedron, 1985, 4, 1625.

24 C. A. Tolman, Chem. Rev., 1977, 77, 313. 